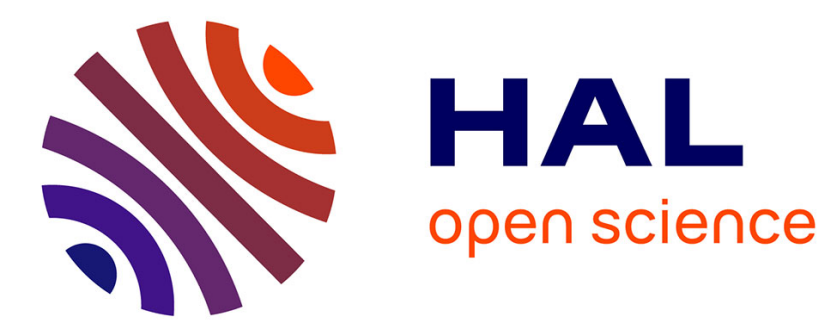

\title{
A Comprehensive Framework for Performance Analysis of Cooperative Multi-Hop Wireless Systems over Log-Normal Fading Channels
}

\author{
Marco Di Renzo, Fabio Graziosi, Fortunato Santucci
}

\section{To cite this version:}

Marco Di Renzo, Fabio Graziosi, Fortunato Santucci. A Comprehensive Framework for Performance Analysis of Cooperative Multi-Hop Wireless Systems over Log-Normal Fading Channels. IEEE Transactions on Communications, 2010, 58 (2), pp. 531-544. hal-00547017

\section{HAL Id: hal-00547017 \\ https://hal.science/hal-00547017}

Submitted on 15 Dec 2010

HAL is a multi-disciplinary open access archive for the deposit and dissemination of scientific research documents, whether they are published or not. The documents may come from teaching and research institutions in France or abroad, or from public or private research centers.
L'archive ouverte pluridisciplinaire HAL, est destinée au dépôt et à la diffusion de documents scientifiques de niveau recherche, publiés ou non, émanant des établissements d'enseignement et de recherche français ou étrangers, des laboratoires publics ou privés. 


\title{
A Comprehensive Framework for Performance Analysis of Cooperative Multi-Hop Wireless Systems over Log-Normal Fading Channels
}

\author{
Marco Di Renzo, Member, IEEE, Fabio Graziosi, Member, IEEE, and Fortunato Santucci, Senior Member, IEEE
}

\begin{abstract}
In this paper, we propose a comprehensive framework for performance analysis of multi-hop multi-branch wireless communication systems over Log-Normal fading channels. The framework allows to estimate the performance of Amplify and Forward (AF) relay methods for both Channel State Information (CSI-) assisted relays, and fixed-gain relays. In particular, the contribution of this paper is twofold: $i)$ first of all, by relying on the Gauss Quadrature Rule (GQR) representation of the Moment Generation Function (MGF) for a Log-Normal distribution, we develop accurate formulas for important performance indexes whose accuracy can be estimated a priori and just depends on GQR numerical integration errors; ii) then, in order to simplify the computational burden of the former framework for some system setups, we propose various approximations, which are based on the Improved Schwartz-Yeh (I-SY) method. We show with numerical and simulation results that the proposed approximations provide a good trade-off between accuracy and complexity for both Selection Combining (SC) and Maximal Ratio Combining (MRC) cooperative diversity methods.
\end{abstract}

Index Terms-Cooperative systems, multi-hop, antenna sharing, spatial diversity, log-normal fading.

\section{INTRODUCTION}

$\mathbf{O}$ VER the last years, spatial diversity techniques have been proved to be a very effective remedy to boost channel capacity and improve error performance over fading channels [1]-[3]. In the recent period, a new concept of spatial diversity is gaining growing attention in the research community: cooperative diversity [4]-[6]. The basic premise of cooperative diversity is to achieve the benefits of spatial diversity without requiring each mobile radio to be equipped with co-located multiple antennas. Instead, each mobile radio becomes part of a large distributed array, and shares its singleantenna to help the communication between two neighboring source and destination radios by using relayed transmissions, and distributed diversity combining techniques [6].

Paper approved by G. Karagiannidis, the Editor for Fading Channels and Diversity of the IEEE Communications Society. Manuscript received June 4, 2008; revised January 25, 2009, May 2, 2009, and July 13, 2009.

This paper was presented in part at the IEEE Global Communications Conference (GLOBECOM), New Orleans, LA, USA, November 2008.

M. Di Renzo is with the French National Center for Scientific Research (CNRS), Laboratory for Signals and Systems (LSS), École Supérieure d'Électricité (SUPÉLEC), 3 rue Joliot-Curie, 91192 Gif-sur-Yvette cedex (Paris), France (e-mail: marco.di.renzo@ieee.org).

F. Graziosi and F. Santucci are with the Department of Electrical and Information Engineering and the Center of Excellence in Research DEWS, University of L'Aquila, 67040 Poggio di Roio, L'Aquila, Italy (e-mail: \{fabio.graziosi, fortunato.santucci\}@univaq.it).

Digital Object Identifier 10.1109/TCOMM.2010.02.080273
Various analytical frameworks have been developed to analyze the performance of virtual antenna array systems over fading channels (see, e.g., [7]-[13] and references therein). However, a careful review of the up-do-date open technical literature has pointed out that these analytical studies are limited to a restricted number of fading channel models, i.e., Rayleigh, Nakagami-m, Nakagami-n (Rice), Nakagamiq (Hoyt), and Weibull. While, the analysis of Log-Normal and composite fading channel models is, to the authors' best knowledge, still very limited [14]-[18]. However, LogNormal fading is often encountered in many reference scenarios of practical interest. For example, it typically characterizes the shadowing effects from indoor obstacles and moving human bodies, and the Log-Normal distribution provides, in general, a better fit for empirical fading channel measurements and for modeling fading fluctuations in indoor radio propagation environments, where long- and short-term contributions tend to get mixed, and the Log-Normal contribution tends to be the dominant factor [19], [20]. Moreover, it is known that shadowing effects in outdoor scenarios are well modeled by a Log-Normal distribution $[1]^{1}$. Finally, and more importantly, recent experimental activities have shown the need to take into account the randomness induced by Log-Normal fading effects for a proper analysis, design, and optimization of cooperative multi-hop networks, as well as pointed out that oversimplifying these wireless propagation phenomena may lead to erroneous protocol and system design guidelines [22][26].

Motivated by the above considerations, in the present contribution we propose a comprehensive framework for the analysis of cooperative multi-hop wireless systems over Log-Normal fading channel, which aims at overcoming the limitations of previous proposed frameworks on the same subject. In particular, the following limitations can be acknowledged in the available contributions: i) in [14], only the Outage Probability $\left(\mathrm{P}_{\text {out }}\right)$ is investigated, and the suggested approximation is not substantiated by numerical simulations, ii) in [15], as the authors are mainly interested in analyzing optimal power allocation issues, and performing diversity gain analysis, Chernoff bounds are used, which, however, may not provide accurate results; furthermore, only the basic dualbranch dual-hop scenario is analyzed therein, and iii) in [16],

\footnotetext{
${ }^{1}$ Note that indoor and outdoor scenarios are both considered, e.g., within the IEEE 802.16j Broadband Wireless Access Working Group [21], which is working on standardization activities for wireless multi-hop networks.
} 
although the proposed framework is very general, explicit closed-form formulas are not provided, but only integral results are available for most performance indexes. Moreover, [14] and [16] only consider the scenario where the relays have full Channel State Information (CSI), while the more practical relay schemes with either average or no CSI are, to date, analyzed only in [15] for Log-Normal fading channels. However, only single-relay networks are investigated therein.

In the light of the above, the contribution of the present paper is twofold: i) first of all, by relying on the Gauss Quadrature Rule (GQR) representation of the Moment Generation Function (MGF) for a Log-Normal distribution, we develop accurate formulas for computing important performance indexes, i.e., Average Bit Error Probability (ABEP), $\mathrm{P}_{\text {out }}$, Outage Capacity (OC), and Ergodic (Shannon) Capacity (EC) for Amplify and Forward (AF) relay methods; ii) then, in order to simplify the computation burden of the former framework for some system setups, we also propose and analyze various approximations, which are based on the recently proposed Improved Schwartz-Yeh (I-SY) method $[27]^{2}$. We show with numerical and simulation results that the proposed approximations provide a good trade-off between accuracy and complexity for both Selection Combining (SC) and Maximal Ratio Combining (MRC) cooperative diversity methods. Various relay schemes, which include CSI-assisted analog relays [7], and semi-blind and blind analog relays [12], will be considered in the analysis.

The remainder of the paper is organized as follows. Section II describes system and channel models. In Section III, the GQR-based framework is presented for SC and MRC methods. In Section IV, various approximations based on the I-SY method are provided and compared. Section V analyzes the computational complexity of the proposed frameworks. Finally, Section VI shows some numerical results to validate the accuracy of them, and Section VI concludes the paper.

Notation. The following notation is used throughout the paper: i) $\log N(\mu, \sigma)$ denotes a $\log -$ Normal Random Variable (RV) with parameters (in $\mathrm{dB}) \mu$ and $\sigma$, ii) $\mathbf{v}(i)$ denotes the $i$-th element of vector $\mathbf{v}$, and $\mathbf{M}(i, j)$ the element in the $i$-th row and $j$-th column of matrix $\mathbf{M}$, iii) $\left\{x_{p}\right\}_{p=1}^{N_{p}}$ and $\left\{H_{x_{p}}\right\}_{p=1}^{N_{p}}$ are zeros and weights of the $N_{p}$-order Hermite polynomial, respectively, iv) $\mathscr{N}_{0}$ is the power spectral density of the Additive White Gaussian Noise (AWGN) of every transceiver and $E_{s}$ is the average radiated energy per transmitted symbol, v) $\operatorname{Pr}\{\cdot\}$ means probability, vi) $Q(x)=$ $(1 / 2) \operatorname{erfc}(x / \sqrt{2})$ is the $Q(\cdot)$ function, and $\operatorname{erfc}(\cdot)$ is the complementary error function in [29, Eq. (7.1.2)], vii) the function $\mathbb{1}_{\{x \leq \epsilon\}}$ is defined as $\mathbb{1}_{\{x \leq \epsilon\}}=1$ if $x \leq \epsilon$, and $\mathbb{1}_{\{x \leq \epsilon\}}=0$ otherwise, viii) $E\{\cdot\}$ denotes statistical expectation, ix) $f_{X}(\cdot)$ and $M_{X}=E\{\exp (-s X)\}$ are the Probability Density Function (PDF) and the MGF of RV X, $\mathrm{x}$ ) $\max \left\{X_{1}, \ldots, X_{n}\right\}$ and $\min \left\{X_{1}, \ldots, X_{n}\right\}$ are the maximum and minimum of RVs $\left\{X_{i}\right\}_{i=1}^{n}$, respectively, xi) $\Gamma(\cdot)$ is the Gamma function in [29, Eq. (6.1.1)], xii) $\delta(\cdot)$ and $\delta_{n, m}$ are Dirac's and Kronecker's Delta functions, respectively, xiii)

\footnotetext{
${ }^{2}$ Recently, a Fenton-Wilkinson based method has also been proposed in [28] to compute the EC for Log-Normal channels, but the accuracy of it has not been tested for cooperative diversity. In this paper we use a different approach, i.e., the I-SY method.
}

$J_{\nu}(\cdot)$ and $I_{\nu}(\cdot)$ are the Bessel and modified Bessel functions of first kind and order $\nu$ in [29, Ch. 9)], respectively, and xiv) $\mathrm{C}(X ; k)=k^{-1} \log _{2}(1+X)$ is the (instantaneous) channel capacity of $\mathrm{RV} \mathrm{X}$.

Moreover, by denoting with RV $X$ the end-to-end Signal-to-Noise Ratio (SNR) of a generic system setup $S$, we define ABEP, $\mathrm{P}_{\text {out }}, \mathrm{EC}$, and $\mathrm{OC}$ as follows, respectively: 1) $\operatorname{ABEP}^{\mathrm{S}}(X ; a, b)=\int_{0}^{+\infty} b Q(a \sqrt{\xi}) f_{X}(\xi) d \xi=$ $(b / \pi) \int_{0}^{\pi / 2} M_{X}\left(0.5 a^{2} / \sin ^{2}(\theta)\right) d \theta$, which holds for linear modulation schemes with coherent detection, $a$ and $b$ are constant factors depending on the modulation scheme, and the last equality is known as Craig's formula, 2) $\mathrm{P}_{\text {out }}^{\mathrm{S}}\left(X ; V_{T}\right)=\int_{0}^{V_{T}} f_{X}(\xi) d \xi$, where $V_{T}$ is the protection threshold for reliable communications, 3) $\operatorname{EC}^{\mathrm{S}}(X ; B)=$ $B^{-1} \int_{0}^{+\infty} \log _{2}(1+\xi) f_{X}(\xi) d \xi$, with $B$ being a scaling factor that depends on the system setup, and 4) $\mathrm{OC}^{\mathrm{S}}(X ; B, r)=$ $\int_{0}^{2^{B r}-1} f_{X}(\xi) d \xi$, where $r$ is the desired rate in [bits/s/Hz].

\section{SySTEM MODEL}

\section{A. Parallel Relay Channel}

Let us consider a typical multi-branch multi-hop cooperative network with $L$ virtual diversity branches and $\left\{N_{l}\right\}_{l=1}^{L}$ hops for every branch, with $M=\sum_{l=1}^{L}\left(N_{l}-1\right)$ representing the total number of relays in the network (see, e.g., [16, Fig. 1] for a similar system setup). In such a network, the communication between a source (S) and a destination (D) is facilitated by $M$ relays $\left\{\left\{R_{l, n}\right\}_{l=1}^{L}\right\}_{n=1}^{N_{l}-1}$, which amplify the signal received by either the source or the previous relay in the same branch, and route the amplified signal to the destination (AF relay technique) [6]. More specifically, i) $R_{l, n}$ denotes the $n$-th relay in the $l$-th branch, and $G_{l, n}$ is the related relay gain, ii) $\alpha_{l, n}$ is the fading amplitude in the $n$-th hop of the $l$-th branch, and $\gamma_{l, n}=\alpha_{l, n}^{2} E_{s} / \mathscr{N}_{0}$ the related SNR, and iii) $\gamma_{0}=\alpha_{0}^{2} E_{s} / \mathscr{N}_{0}$ denotes the SNR of the direct path between $\mathrm{S}$ and D (i.e., one-hop transmission). Moreover, the normalized SNR $\tilde{\gamma}_{l, n}=\gamma_{l, n} /\left(E_{s} / \mathscr{N}_{0}\right)=\alpha_{l, n}^{2}$ will be assumed to be Log-Normal distributed with parameters (in dB) $\mu_{l, n}$ and $\sigma_{l, n}$, i.e.,:

$f_{\tilde{\gamma}_{l, n}}(\xi)=\frac{10}{\ln (10)} \frac{1}{\sqrt{2 \pi} \sigma_{l, n} \xi} \exp \left[-\frac{\left(10 \log _{10}(\xi)-\mu_{l, n}\right)^{2}}{2 \sigma_{l, n}^{2}}\right]$

Depending on the a priori CSI knowledge at the relays, the end-to-end SNR in every diversity branch, $\gamma_{l}$, may take one of the following forms:

1) Full CSI (F-CSI), i.e., CSI-assisted analog relays, [5],

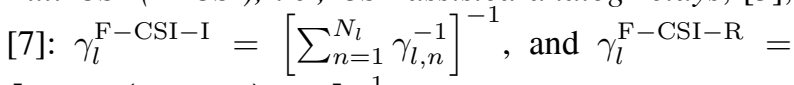
$\left[\prod_{n=1}^{N_{l}}\left(1+\gamma_{l, n}^{-1}\right)-1\right]^{-1}$, which are obtained by set$\operatorname{ting} G_{l, n}=1 / \alpha_{l, n}$ and $G_{l, n}=\sqrt{1 /\left(\alpha_{l, n}^{2}+\mathscr{N}_{0} / E_{s}\right)}$, respectively. When the $\mathrm{F}-\mathrm{CSI}-\mathrm{I}$ setup is considered, it is assumed that every relay can invert the fading effect of the previous hop without imposing any limits on the output power of the relay. On the other hand, the FCSI-R system setup limits the output power of every 


$$
\left\{\begin{array}{l}
\Pi_{l}(\mathbf{p})=\prod_{k=1}^{N_{l}} \frac{H_{p_{k}}}{\sqrt{\pi}} \\
\Omega_{l}(\mathbf{p})=\sum_{k=1}^{N_{l}} \exp \left[\frac{\ln (10)}{10} \sqrt{2} \sum_{j=1}^{N_{l}} \varsigma_{l}(k, j) x_{p_{j}}+\frac{\ln (10)}{10} \boldsymbol{\mu}_{l}(k)\right]
\end{array}\right.
$$

relay if the fading amplitude of the previous hop is too low.

2) No CSI (N-CSI), i.e., blind analog relays, [12]: $\gamma_{l}^{\mathrm{N}-\mathrm{CSI}}=\left[\sum_{n=1}^{N_{l}}\left(\prod_{j=1}^{n} C_{l, j-1} \gamma_{l, j}^{-1}\right)\right]^{-1}$, where $G_{l, j}=\sqrt{E_{s} /\left(C_{l, j} \mathscr{N}_{0}\right)}$ and $\left\{C_{l, 0}\right\}_{l=1}^{L}=1$.

3) Average CSI (A-CSI), i.e., semi-blind analog relays, [12]: The end-to-end SNR is formally the same as the blind scenario, i.e., $\gamma_{l}^{\mathrm{A}-\mathrm{CSI}}=\gamma_{l}^{\mathrm{N}-\mathrm{CSI}}$, but the relay gain is $G_{l, n}^{2}=E\left\{\left(\alpha_{l, n}^{2}+\mathscr{N}_{0} / E_{s}\right)^{-1}\right\}$. For LogNormal fading channels, it can be obtained by putting the GQR-closed-form expression of the MGF of a LogNormal distribution [2, Eq. (2.28)] into [30, Eq. (4.1)], and computing the related integral as follows:

$$
G_{l, n}^{2}=\frac{1}{\sqrt{\pi}} \sum_{p=1}^{N_{p}} \frac{H_{x_{p}}}{10\left[\left(\sqrt{2} \sigma_{l, n} x_{p}+\mu_{l, n}\right) / 10\right]+\mathscr{N}_{0} / E_{s}}
$$

where $\left\{x_{p}\right\}_{p=1}^{N_{p}}$ and $\left\{H_{x_{p}}\right\}_{p=1}^{N_{p}}$ can be found in [29, Table 25.10]. The interested reader may refer to [31] for issues related to truncation errors and convergence conditions of GQR integration.

With regard to diversity combining techniques, we analyze the performance of SC and MRC methods, whose end-toend SNRs at the combiner output are given by $\gamma_{S C}=$ $\max \left\{\gamma_{0}, \gamma_{1}, \ldots, \gamma_{L}\right\}$ and $\gamma_{M R C}=\sum_{l=0}^{L} \gamma_{l}$, respectively. Moreover, for analytical tractability, but without loss of generality, in what follows the direct path $\gamma_{0}$ will be implicitly treated as a multi-hop link with a single hop, i.e., $N_{0}=1$.

\section{B. Performance Analysis: The Need of Modeling Log-Normal Power-Sums}

By carefully looking at $\gamma_{l}^{\mathrm{F}-\mathrm{CSI}-\mathrm{R}}, \gamma_{l}^{\mathrm{F}-\mathrm{CSI}-\mathrm{I}}, \gamma_{l}^{\mathrm{N}-\mathrm{CSI}}$, and $\gamma_{l}^{\mathrm{A}-\mathrm{CSI}}$, we can easily figure out that, regardless of the a priori knowledge of relay's CSI, the inverse of the SNR of every diversity branch is given by the summation of correlated Log-Normal RVs. In particular, these SNRs can be written as $\gamma_{l}=\left[\sum_{n=1}^{N_{l}} Y_{l, n}\right]^{-1}$, where $\left\{Y_{l, n}\right\}_{n=1}^{N_{l}}$ are Log-Normal RVs with mean vector $\left(\boldsymbol{\mu}_{Y_{l}}\right)$ and covariance matrix $\left(\boldsymbol{\Sigma}_{Y_{l}}\right)$ given as follows:

1) $\gamma_{l}^{\mathrm{F}-\mathrm{CSI}-\mathrm{I}}: \boldsymbol{\mu}_{Y_{l}}(n)=-\mu_{l, n}-10 \log _{10}\left(E_{s} / \mathscr{N}_{0}\right)$, and $\Sigma_{Y_{l}}(n, m)=\sigma_{l, n}^{2} \delta_{n, m}$

2) $\gamma_{l}^{\mathrm{N}-\mathrm{CSI}}$ and $\gamma_{l}^{\mathrm{A}-\mathrm{CSI}}: \boldsymbol{\Sigma}_{Y_{l}}(n, m)=\sum_{j=1}^{\min (n, m)} \sigma_{l, j}^{2}$, and $\boldsymbol{\mu}_{Y_{l}}(n)=\sum_{j=1}^{n}\left(-\mu_{l, j}-10 \log _{10}\left(G_{l, j}^{2}\right)\right)$;

3) $\gamma_{l}^{\mathrm{F}-\mathrm{CSI}-\mathrm{R}}$ : see Appendix I.

Accordingly, modeling the distribution of these SNRs is equivalent to find the distribution of the power-sum of generically correlated Log-Normal RVs. However, a closed-form solution for the PDF of such a power-sum is still unknown to date, even though several approximation techniques have been proposed to deal with the above problem [27]. Moreover, although closed-form solutions for the PDF do not exist, an efficient and accurate representation via GQR methods is available in the literature to compute the MGF [32]. In the present paper, we will move from and generalize these results for performance analysis of cooperative multi-hop networks.

Finally, we would like to emphasize that in the present contribution we need to model the power-sum of correlated Log-Normal RVs as a consequence of the particular structure of the end-to-end SNRs. In other words, even though fading effects can be assumed to be uncorrelated, computing the endto-end performance of multi-hop networks needs to develop frameworks that allow to deal with correlated summands. So, even though we resort to the assumption of uncorrelated fading in this manuscript, the proposed frameworks can be used to analyze the performance of multi-hop networks over generically correlated fading environments. The generalization to scenarios with correlated fading among the diversity branches is, on the contrary, left to a future contribution.

\section{Accurate Framework for Performance ANALYSIS}

\section{A. Multi-Hop Networks - No Diversity}

Although the PDF of $\gamma_{l}^{-1}$ is not available in closed-form, its MGF can be computed as follows [32]:

$$
M_{\gamma_{l}^{-1}}(s)=\sum_{p_{1}=1}^{N_{p}} \sum_{p_{2}=1}^{N_{p}} \cdots \sum_{p_{N_{l}}=1}^{N_{p}}\left[\Pi_{l}(\mathbf{p}) e^{-s \Omega_{l}(\mathbf{p})}\right]
$$

with $\Pi_{l}(\cdot)$ and $\Omega_{l}(\cdot)$ being defined in (4) on top of this page, and $\varsigma(k, j)$ is the $(k, j)$-th element of $\boldsymbol{\Sigma}_{Y_{l}}^{s q}=\boldsymbol{U}_{Y_{l}}\left(\mathbf{V}_{Y_{l}}\right)^{0.5}$, $\boldsymbol{U}_{Y_{l}}$ and $\boldsymbol{V}_{Y_{l}}$ are the matrices containing eigenvectors and eigenvalues of $\boldsymbol{\Sigma}_{Y_{l}}$, and $\mathbf{p}$ is a vector with elements $\left\{p_{j}\right\}_{j=1}^{N_{l}}$.

From (3), the MGF of $\gamma_{l}$ can be obtained using the recent result [16, Theorem 1]:

$$
M_{\gamma_{l}}(s)=1-2 \sqrt{s} \int_{0}^{+\infty} J_{1}(2 \sqrt{s} \xi) M_{\gamma_{l}^{-1}}\left(\xi^{2}\right) d \xi
$$

which can be solved using GQR integration and [33, Eq. 6.618], as shown in (6) on top of the next page, where $\stackrel{(a)}{=}$ and $\stackrel{(b)}{=}$ are obtained by using [29, Eq. (10.2.13)] and [29, Eq. (4.5.1)], respectively. Moreover, $\stackrel{(c)}{=}$ comes from the identity $\sum_{p_{1}=1}^{N_{p}} \sum_{p_{2}=1}^{N_{p}} \cdots \sum_{p_{N_{l}}=1}^{N_{p}} \Pi_{l}(\mathbf{p})=1$.

Finally, via inverse Laplace transform [34], the PDF, $f_{\gamma_{l}}(\cdot)$, is as follows:

$$
f_{\gamma_{l}}(\xi)=\sum_{p_{1}=1}^{N_{p}} \sum_{p_{2}=1}^{N_{p}} \cdots \sum_{p_{N_{l}}=1}^{N_{p}}\left[\Pi_{l}(\mathbf{p}) \delta\left(\xi-\frac{1}{\Omega_{l}(\mathbf{p})}\right)\right]
$$




$$
\begin{aligned}
M_{\gamma_{l}}(s) & =1-\sqrt{\pi s} \sum_{p_{1}=1}^{N_{p}} \sum_{p_{2}=1}^{N_{p}} \cdots \sum_{p_{N_{l}}=1}^{N_{p}}\left[\frac{\Pi_{l}(\mathbf{p})}{\sqrt{\Omega_{l}(\mathbf{p})}} \exp \left(-\frac{s}{2 \Omega_{l}(\mathbf{p})}\right) I_{\frac{1}{2}}\left(\frac{s}{2 \Omega_{l}(\mathbf{p})}\right)\right] \\
& \stackrel{(a)}{=} 1-2 \sum_{p_{1}=1}^{N_{p}} \sum_{p_{2}=1}^{N_{p}} \cdots \sum_{p_{N_{l}}=1}^{N_{p}}\left[\Pi_{l}(\mathbf{p}) \exp \left(-\frac{s}{2 \Omega_{l}(\mathbf{p})}\right) \sinh \left(\frac{s}{2 \Omega_{l}(\mathbf{p})}\right)\right] \\
& \stackrel{(b)}{=} 1-\sum_{p_{1}=1}^{N_{p}} \sum_{p_{2}=1}^{N_{p}} \cdots \sum_{p_{N_{l}}=1}^{N_{p}}\left\{\Pi_{l}(\mathbf{p})\left[1-\exp \left(-\frac{s}{\Omega_{l}(\mathbf{p})}\right)\right]\right\} \stackrel{(c)}{=} \sum_{p_{1}=1}^{N_{p}} \sum_{p_{2}=1}^{N_{p}} \cdots \sum_{p_{N_{l}}=1}^{N_{p}}\left[\Pi_{l}(\mathbf{p}) \exp \left(-\frac{s}{\Omega_{l}(\mathbf{p})}\right)\right]
\end{aligned}
$$

$$
\operatorname{ABEP}^{\mathrm{SC}}\left(\gamma_{S C} ; a, b\right)=b \sum_{l=0}^{L} \sum_{p_{1}=1}^{N_{p}} \sum_{p_{2}=1}^{N_{p}} \cdots \sum_{p_{N_{l}}=1}^{N_{p}}\left[\Pi_{l}(\mathbf{p}) Q\left(a \sqrt{\frac{1}{\Omega_{l}(\mathbf{p})}}\right) \prod_{k=0}^{L} \mathrm{P}_{\text {out }}^{\mathrm{Mh}}\left(\gamma_{k \neq l} ; \frac{1}{\Omega_{k}(\mathbf{p})}\right)\right]
$$

$$
\operatorname{EC}^{\mathrm{SC}}\left(\gamma_{S C} ; M+1\right)=\frac{1}{(M+1)} \sum_{l=0}^{L} \sum_{p_{1}=1}^{N_{p}} \sum_{p_{2}=1}^{N_{p}} \cdots \sum_{p_{N_{l}}=1}^{N_{p}}\left[\Pi_{l}(\mathbf{p}) \log _{2}\left(1+\frac{1}{\Omega_{l}(\mathbf{p})}\right) \prod_{k=0}^{L} \mathrm{P}_{\text {out }}^{\mathrm{Mh}}\left(\gamma_{k \neq l} ; \frac{1}{\Omega_{k}(\mathbf{p})}\right)\right]
$$

It is possible to verify that (7) is a true PDF as the following two conditions are satisfied simultaneously: i) $f_{\gamma_{l}}(\cdot)$ is positive semi-definite for each value of its argument, and ii) $\int_{0}^{+\infty} f_{\gamma_{l}}(\xi) d \xi=1$. The first condition can be proved by taking into account that, by definition, the GQR weights are always positive. The second condition can be obtained by taking into account that $\int_{0}^{+\infty} \delta\left(\xi-\Omega_{l}^{-1}(\mathbf{p})\right) d \xi=1$ and $\sum_{p_{1}=1}^{N_{p}} \sum_{p_{2}=1}^{N_{p}} \cdots \sum_{p_{N_{l}}=1}^{N_{p}} \Pi_{l}(\mathbf{p})=1$. Similar arguments can be used to verify that other PDFs developed in this paper are true PDFs.

Therefore, by using (7), $\mathrm{ABEP}, \mathrm{P}_{\text {out }}$, and $\mathrm{EC}^{3}$ for multihop (Mh) systems over Log-Normal fading channels can be obtained via simple algebraic manipulations, as follows:

$$
\begin{aligned}
& \operatorname{ABEP}^{\mathrm{Mh}}\left(\gamma_{l} ; a, b\right) \\
& \quad=b \sum_{p_{1}=1}^{N_{p}} \sum_{p_{2}=1}^{N_{p}} \cdots \sum_{p_{N_{l}}=1}^{N_{p}}\left[\Pi_{l}(\mathbf{p}) Q\left(a \sqrt{\frac{1}{\Omega_{l}(\mathbf{p})}}\right)\right] \\
& \mathrm{P}_{\text {out }}^{\mathrm{Mh}}\left(\gamma_{l} ; V_{T}\right) \\
& \quad=\sum_{p_{1}=1}^{N_{p}} \sum_{p_{2}=1}^{N_{p}} \cdots \sum_{p_{N_{l}}=1}^{N_{p}}\left[\Pi_{l}(\mathbf{p}) \cdot \mathbb{1}_{\left\{\Omega_{l}^{-1}\left(\mathbf{p}_{l}\right) \leq V_{T}\right\}}\right] \\
& \mathrm{EC}^{\mathrm{Mh}}\left(\gamma_{l} ; N_{l}\right) \\
& =\frac{1}{N_{l}} \sum_{p_{1}=1}^{N_{p}} \sum_{p_{2}=1}^{N_{p}} \cdots \sum_{p_{N_{l}}=1}^{N_{p}}\left[\Pi_{l}(\mathbf{p}) \log _{2}\left(1+\frac{1}{\Omega_{l}(\mathbf{p})}\right)\right]
\end{aligned}
$$

Note that the scaling factor $B=N_{l}$ in EC is due to the assumption of a time division channel allocation scheme for delivering information through the network [35], [36]. When SC and MRC diversity is considered the scaling factor is $B=M+1$, with $M$ defined in Section II. Finally, $\mathrm{OC}^{\mathrm{Mh}}\left(\gamma_{l} ; N_{l}, r\right)=\mathrm{P}_{\text {out }}^{\mathrm{Mh}}\left(\gamma_{l} ; V_{T}=2^{r N_{l}}-1\right)$.

\footnotetext{
${ }^{3}$ Note that, similar to [35], [36], the expression for computing channel capacity neglects the effect of discontinuous transmissions among the diversity branches of the network, and delay transmission of bits in every relay node of it.
}

\section{B. Multi-Branch Multi-Hop Networks - Distributed Diversity}

1) Selection Combining ( $S C$ ): By assuming that the signals received in every diversity branch are independent from each other, $\mathrm{P}_{\text {out }}$ for $\mathrm{SC}$ methods is given by:

$$
\begin{aligned}
\mathrm{P}_{\text {out }}^{\mathrm{SC}}\left(\gamma_{S C} ; V_{T}\right) & =\operatorname{Pr}\left\{\gamma_{S C} \leq V_{T}\right\} \\
& =\operatorname{Pr}\left\{\max \left\{\gamma_{0}, \gamma_{1}, \ldots, \gamma_{L}\right\} \leq V_{T}\right\} \\
& =\operatorname{Pr}\left\{\gamma_{0} \leq V_{T}, \gamma_{1} \leq V_{T}, \ldots, \gamma_{L} \leq V_{T}\right\} \\
& =\prod_{l=0}^{L} \operatorname{Pr}\left\{\gamma_{l} \leq V_{T}\right\}=\prod_{l=0}^{L} \mathrm{P}_{\text {out }}^{\mathrm{Mh}}\left(\gamma_{l} ; V_{T}\right)
\end{aligned}
$$

From (11), the PDF of $\gamma_{S C}$ can be obtained via simple differentiation:

$$
\begin{aligned}
f_{\gamma_{S C}}(\xi) & =\frac{d}{d \xi}\left[\prod_{l=0}^{L} \mathrm{P}_{\text {out }}^{\mathrm{Mh}}\left(\gamma_{l} ; \xi\right)\right] \\
& =\sum_{l=0}^{L}\left[f_{\gamma_{l}}(\xi) \prod_{k=0}^{L} \mathrm{P}_{\text {out }}^{\mathrm{Mh}}\left(\gamma_{k \neq l} ; \xi\right)\right]
\end{aligned}
$$

Furthermore, from (12), ABEP and EC can be computed as as shown in (13) and (14) on top of this page, respectively.

2) Maximal Ratio Combining (MRC): By still assuming that the signals received in every diversity branch are independent from each other, the MGF for MRC diversity can be written as shown in (15) on top of the next page. From (15), the PDF of $\gamma_{M R C}$ is computed via inverse Laplace transform operations as shown in (16) on top of the next page. Furthermore, ABEP, $\mathrm{P}_{\text {out }}$, and EC are shown in (17)-(19) on top of the next page, respectively.

Although, for the sake of conciseness, just SC and MRC combining schemes are here analyzed, the reader may use a similar development for other diversity methods [2].

\section{I-SY Method For Performance AnAlysis}

The framework developed in Section III provides very accurate results, and for most system setups it represents a 


$$
\begin{aligned}
M_{\gamma_{M R C}}(s) & =\prod_{l=0}^{L} M_{\gamma_{l}}(s)=\prod_{l=0}^{L}\left\{\sum_{p_{1, l}=1}^{N_{p}} \sum_{p_{2, l}=1}^{N_{p}} \cdots \sum_{p_{N_{l}, l}=1}^{N_{p}}\left[\Pi_{l}\left(\mathbf{p}_{l}\right) \exp \left(-\frac{s}{\Omega_{l}\left(\mathbf{p}_{l}\right)}\right)\right]\right\} \\
& =\sum_{p_{1,0}=1}^{N_{p}} \cdots \sum_{p_{N_{0}, 0}=1}^{N_{p}} \cdots \sum_{p_{1, L}=1}^{N_{p}} \cdots \sum_{p_{N_{L}, L}=1}^{N_{p}}\left\{\left[\prod_{l=0}^{L} \Pi_{l}\left(\mathbf{p}_{l}\right)\right] \exp \left(-s \sum_{l=0}^{L} \frac{1}{\Omega_{l}\left(\mathbf{p}_{l}\right)}\right)\right\}
\end{aligned}
$$

$$
f_{\gamma_{M R C}}(\xi)=\sum_{p_{1,0}=1}^{N_{p}} \ldots \sum_{p_{N_{0}, 0}=1}^{N_{p}} \ldots \sum_{p_{1, L}=1}^{N_{p}} \ldots \sum_{p_{N_{L}, L}=1}^{N_{p}}\left\{\left[\prod_{l=0}^{L} \Pi_{l}\left(\mathbf{p}_{l}\right)\right] \delta\left(\xi-\sum_{l=0}^{L} \frac{1}{\Omega_{l}\left(\mathbf{p}_{l}\right)}\right)\right\}
$$

$$
\operatorname{ABEP}^{\mathrm{MRC}}\left(\gamma_{M R C} ; a, b\right)=b \sum_{p_{1,0}=1}^{N_{p}} \cdots \sum_{p_{N_{0}, 0}=1}^{N_{p}} \cdots \sum_{p_{1, L}=1}^{N_{p}} \ldots \sum_{p_{N_{L}, L}=1}^{N_{p}}\left\{\left[\prod_{l=0}^{L} \Pi_{l}\left(\mathbf{p}_{l}\right)\right] Q\left(a \sqrt{\sum_{l=0}^{L} \frac{1}{\Omega_{l}\left(\mathbf{p}_{l}\right)}}\right)\right\}
$$

$$
\mathrm{P}_{\text {out }}^{\mathrm{MRC}}\left(\gamma_{M R C} ; V_{T}\right)=\sum_{p_{1,0}=1}^{N_{p}} \ldots \sum_{p_{N_{0}, 0}=1}^{N_{p}} \cdots \sum_{p_{1, L}=1}^{N_{p}} \ldots \sum_{p_{N_{L}, L}=1}^{N_{p}}\left\{\left[\prod_{l=0}^{L} \Pi_{l}\left(\mathbf{p}_{l}\right)\right] \cdot \mathbb{1}_{\left\{\sum_{l=0}^{L} \Omega_{l}^{-1}\left(\mathbf{p}_{l}\right) \leq V_{T}\right\}}\right\}
$$

$$
\operatorname{EC}^{\mathrm{MRC}}\left(\gamma_{M R C} ; M+1\right)=\frac{1}{M+1} \sum_{p_{1,0}=1}^{N_{p}} \ldots \sum_{p_{N_{0}, 0}=1}^{N_{p}} \ldots \sum_{p_{1, L}=1}^{N_{p}} \ldots \sum_{p_{N_{L}, L}=1}^{N_{p}}\left\{\left[\prod_{l=0}^{L} \Pi_{l}\left(\mathbf{p}_{l}\right)\right] \log _{2}\left(1+\sum_{l=0}^{L} \frac{1}{\Omega_{l}\left(\mathbf{p}_{l}\right)}\right)\right\}
$$

$$
\operatorname{ABEP}^{\mathrm{SC}, \mathrm{I}-\mathrm{SY}}\left(\gamma_{S C} ; a, b\right) \cong \frac{b}{\sqrt{\pi}} \sum_{l=0}^{L} \sum_{p=1}^{N_{p}}\left\{H_{p} Q\left(a \sqrt{10^{\left(\sqrt{2} \tilde{\sigma}_{Y_{l}} x_{p}-\tilde{\mu}_{Y_{l}}\right) / 10}}\right)\left[Q\left(\sqrt{2} x_{p}\right)\right]^{L}\right\}
$$

$$
\operatorname{EC}^{\mathrm{SC}, \mathrm{I}-\mathrm{SY}}\left(\gamma_{S C} ; M+1\right) \cong \frac{1}{\sqrt{\pi}(M+1)} \sum_{l=0}^{L} \sum_{p=1}^{N_{p}}\left\{H_{p} \log _{2}\left(1+10^{\left(\sqrt{2} \tilde{\sigma}_{Y_{l}} x_{p}-\tilde{\mu}_{Y_{l}}\right) / 10}\right)\left[Q\left(\sqrt{2} x_{p}\right)\right]^{L}\right\}
$$

$$
M_{\gamma_{M R C}}^{\mathrm{I}-\mathrm{SY}}(s) \cong\left(\frac{1}{\sqrt{\pi}}\right)^{L+1} \sum_{p_{0}=1}^{N_{p}} \sum_{p_{1}=1}^{N_{p}} \cdots \sum_{p_{L}=1}^{N_{p}}\left[\left(\prod_{l=0}^{L} H_{p_{l}}\right) \exp \left(-s \sum_{l=0}^{L} 10^{\left(\sqrt{2} \tilde{\sigma}_{Y_{l}} x_{p_{l}}-\tilde{\mu}_{Y_{l}}\right) / 10}\right)\right]
$$

very flexible and accurate means for performance analysis and system optimization (e.g., for routing optimization based on $\mathrm{ABEP}, \mathrm{P}_{\text {out }}$, or EC cost functions, and for optimal power allocation of relays). However, it also shows some limitations, which may ask for a simpler solution to be computed and used for system optimization, and which, even being less accurate than the framework in Section III, may still be reasonably accurate in the vast majority of system setups. For example, the framework in Section III may suffer these problems: i) the computation of $\mathrm{P}_{\text {out }}$ (see, e.g., (9)) involves a function (i.e., $\mathbb{1}_{\{\cdot\}}$ ) that is not smooth, and may require a large number (e.g., $>100$ ) of points $N_{p}$ to provide accurate results, ii) when SC diversity is concerned, $L$ terms related to $\mathrm{P}_{\text {out }}$ have to be computed, which may make the framework computational demanding, and iii) when MRC diversity is considered, the framework may be as computational complex as the SC case due to the number of fold summations involved in the computation. So, for those scenarios (typically when both the number of hops and diversity branches is large) where the computational complexity of the framework in Section III is high, we propose in this section various approximations with a different complexity, which may be used as an alternative to it.

The approximations proposed in this section are all based on a method that we call, in this contribution, Improved Schwartz-Yeh (I-SY) approximation. This approach has been recently proposed, as a byproduct of the analysis, in [27], which aimed at considering more complicated (i.e., nonLog-Normal) and accurate approximation techniques for LogNormal power-sum (i.e., Pearson Type IV approximation) ${ }^{4}$. In particular, the method retains the main features of SY approach [37], i.e., i) the Log-Normal power-sum is ap-

\footnotetext{
${ }^{4}$ In this contribution, we do not consider these advanced and non-LogNormal methods because the I-SY method already offers good accuracies, and the required flexibility to elaborate further the obtained approximating formulas for system optimization.
} 
proximated by a Log-Normal RV, and ii) the parameters of the approximating distribution are obtained via a moment matching in the logarithmic domain. However, it shows an important difference: the log-moments are obtained without resorting to recursive numerical methods (in SY method they are obtained via a recursive Log-Normal approximation, which introduces errors in every recursive step). We use this approach to develop several approximations with different complexities and accuracies. Given that in [27] the I-SY method has been proposed for independent summands, in Appendix II we summarize the main steps to generalize it to the power-sum of correlated $\mathrm{Log}-\mathrm{Normal} \mathrm{RVs}^{5}$.

\section{A. Multi-Hop Networks - No Diversity}

For multi-hop systems, the I-SY method foresees to approximate the power sum $Y_{l}=\sum_{n=1}^{N_{l}} Y_{l, n}$, i.e., the inverse of a SNR, with a $\log -$ Normal RV, i.e., $Y_{l} \sim \log N\left(\tilde{\mu}_{Y_{l}}, \tilde{\sigma}_{Y_{l}}\right)$, where $\tilde{\mu}_{Y_{l}}$ and $\tilde{\sigma}_{Y_{l}}$ are the parameters (in $\mathrm{dB}$ ) of the approximating PDF. Using [27] and Appendix II, the latter parameters are $\tilde{\mu}_{Y_{l}}=\tilde{m}_{Y_{l}}^{(1)}$ and $\tilde{\sigma}_{Y_{l}}=\sqrt{\tilde{m}_{Y_{l}}^{(2)}-\left(\tilde{m}_{Y_{l}}^{(1)}\right)^{2}}$, where:

$$
\tilde{m}_{Y_{l}}^{(q)}=\left(\frac{10}{\ln (10)}\right)^{q} \sum_{p_{1}=1}^{N_{p}} \sum_{p_{2}=1}^{N_{p}} \cdots \sum_{p_{N_{l}}=1}^{N_{p}} \Pi_{l}(\mathbf{p})\left\{\ln \left[\Omega_{l}(\mathbf{p})\right]\right\}^{q}
$$

Thus, the end-to-end SNR $\gamma_{l}$ can be approximated with a Log-Normal distribution (when $l=0$ the SNR is actually Log-Normal distributed), i.e., $\gamma_{l} \sim \log N\left(-\tilde{\mu}_{Y_{l}}, \tilde{\sigma}_{Y_{l}}\right)$. In what follows, we denote by $f_{\gamma_{l}}^{\mathrm{I}-\mathrm{SY}}(\cdot)$ this approximating PDF. By relying on the I-SY method, ABEP, $\mathrm{P}_{\text {out }}$ and $\mathrm{EC}$ are as follows:

$$
\begin{aligned}
& \operatorname{ABEP}^{\mathrm{Mh}, \mathrm{I}-\mathrm{SY}}\left(\gamma_{l} ; a, b\right) \\
& \cong \frac{b}{\sqrt{\pi}} \sum_{p=1}^{N_{p}} H_{p} Q\left(a \sqrt{10^{\left(\sqrt{2} \tilde{\sigma}_{Y_{l}} x_{p}-\tilde{\mu}_{Y_{l}}\right) / 10}}\right) \\
& \mathrm{P}_{\mathrm{out}}^{\mathrm{Mh}, \mathrm{I}-\mathrm{SY}}\left(\gamma_{l} ; V_{T}\right) \cong Q\left(-\frac{10 \log _{10}\left(V_{T}\right)+\tilde{\mu}_{Y_{l}}}{\tilde{\sigma}_{Y_{l}}}\right) \\
& \mathrm{EC}^{\mathrm{Mh}, \mathrm{I}-\mathrm{SY}}\left(\gamma_{l} ; N_{l}\right) \\
& \cong \frac{1}{\sqrt{\pi} N_{l}} \sum_{p=1}^{N_{p}} H_{p} \log _{2}\left(1+10^{\left(\sqrt{2} \tilde{\sigma}_{Y_{l}} x_{p}-\tilde{\mu}_{Y_{l}}\right) / 10}\right)
\end{aligned}
$$

By carefully looking at the obtained results, we observe that, in this case, computing $\mathrm{P}_{\text {out }}$ is very simple, and the numerical problems described above have disappeared.

\footnotetext{
${ }^{5}$ Note that a comprehensive analysis of the best Log-Normal or nonLog-Normal approximation method for approximating the power-sum of un-correlated and correlated Log-Normal RVs in the context of cooperative multi-hop wireless systems is out of the scope of the present contribution. The aim of the paper is to show that a particular approximation method (i.e., the I-SY) has the desired degree of flexibility and accuracy to be used in this context. The comparison of various approximation approaches, and the analysis of their advantages and disadvantages is left to a future contribution.
}

\section{B. Multi-Branch Multi-Hop Networks - Selection Combining}

The I-SY approximation can be extended to SC diversity. In particular, $\mathrm{P}_{\text {out }}$ and $f_{\gamma_{S C}}^{\mathrm{I}-\mathrm{SY}}(\cdot)$ are:

$$
\begin{aligned}
& \mathrm{P}_{\text {out }}^{\mathrm{SC}, \mathrm{I}-\mathrm{SY}}\left(\gamma_{S C} ; V_{T}\right) \cong \prod_{l=0}^{L} \mathrm{P}_{\mathrm{out}}^{\mathrm{Mh}, \mathrm{I}-\mathrm{SY}}\left(\gamma_{l} ; V_{T}\right) \\
&=\prod_{l=0}^{L} Q\left(-\frac{10 \log _{10}\left(V_{T}\right)+\tilde{\mu}_{Y_{l}}}{\tilde{\sigma}_{Y_{l}}}\right) \\
& f_{\gamma_{S C}}^{\mathrm{I}-\mathrm{SY}}(\xi) \cong \frac{d}{d \xi}\left[\prod_{l=0}^{L} \mathrm{P}_{\mathrm{out}}^{\mathrm{Mh}, \mathrm{I}-\mathrm{SY}}\left(\gamma_{l} ; \xi\right)\right] \\
&=\sum_{l=0}^{L}\left[f_{\gamma_{l}}^{\mathrm{I}-\mathrm{SY}}(\xi) \prod_{k=0}^{L} \mathrm{P}_{\mathrm{out}}^{\mathrm{Mh}, \mathrm{I}-\mathrm{SY}}\left(\gamma_{k \neq l} ; \xi\right)\right]
\end{aligned}
$$

Then, by using (25), ABEP and EC can be obtained with simple algebraic manipulations as shown in (26) and (27) on top of the previous page, respectively.

\section{Multi-Branch Multi-Hop Networks - Maximal Ratio Com- bining}

When the I-SY method is used in multi-branch multi-hop relay networks with MRC diversity, there exist several possibilities to use it, each one leading to a different framework with its own accuracy and complexity. The aim of this subsection is to analyze various possibilities, and compare them in terms of complexity (see Section V) and accuracy (see Section VI).

1) Maximal Ratio Combining - Method 1: The first method foresees two main steps: i) first of all, $\gamma_{l}$ is approximated with a Log-Normal RV using the I-SY method, i.e., $\gamma_{l} \sim$ $\log N\left(-\tilde{\mu}_{Y_{l}}, \tilde{\sigma}_{Y_{l}}\right)$, and ii) then, ABEP, $\mathrm{P}_{\text {out }}$ and EC are computed using the MGF-based approach for performance analysis of digital communication systems [2].

Accordingly, by relying on the Log-Normal approximation for $\gamma_{l}$, its MGF is [2]:

$$
M_{\gamma_{l}}^{\mathrm{I}-\mathrm{SY}}(s) \cong \frac{1}{\sqrt{\pi}} \sum_{p=1}^{N_{p}} H_{p} \exp \left(-10^{\left(\sqrt{2} \tilde{\sigma}_{Y_{l}} x_{p}-\tilde{\mu}_{Y_{l}}\right) / 10} s\right)
$$

and, the MGF of $\gamma_{M R C}$ becomes $M_{\gamma_{M R C}}^{\mathrm{I}-\mathrm{SY}}(s) \cong$ $\prod_{l=0}^{L} M_{\gamma_{l}}^{\mathrm{I}-\mathrm{SY}}(s)$ shown in (29) on top of the previous page.

Using (29), the ABEP can be computed using the Craig's formula [2] as shown in (30) on top of the next page. Similarly, $\mathrm{P}_{\text {out }}$ can be computed using the Euler-Sum based framework described in [2], whose final expression is not reported here for the sake of conciseness. While, to the authors' best knowledge, it does not exist to date a general formula to compute EC, which exploits the MGF-based approach developed in [2]. However, in Theorem 1 we propose a new and general result that can be used to compute EC from the MGF of the endto-end SNR.

Theorem 1: Let the MGF of a positive RV $X$, the MGF of RV $Y=\log _{2}(1+X)$ is given by the following integral 


$$
\operatorname{ABEP}^{\mathrm{MRC}, \mathrm{I}-\mathrm{SY}}\left(\gamma_{M R C} ; a, b\right) \cong\left(\frac{1}{\sqrt{\pi}}\right)^{L+1} b \sum_{p_{0}=1}^{N_{p}} \sum_{p_{1}=1}^{N_{p}} \cdots \sum_{p_{L}=1}^{N_{p}}\left[( \prod _ { l = 0 } ^ { L } H _ { p _ { l } } ) Q \left(a \sqrt{\left.\left.\sum_{l=0}^{L} 10^{\left(\sqrt{2} \tilde{\sigma}_{Y_{l}} x_{p_{l}}-\tilde{\mu}_{Y_{l}}\right) / 10}\right)\right]}\right.\right.
$$

$$
\mathrm{EC}^{\mathrm{MRC}, \mathrm{I}-\mathrm{SY}}\left(\gamma_{M R C} ; M+1\right) \cong \frac{1}{M+1}\left(\frac{1}{\sqrt{\pi}}\right)^{L+1} \sum_{p_{0}=1}^{N_{p}} \sum_{p_{1}=1}^{N_{p}} \cdots \sum_{p_{L}=1}^{N_{p}}\left[\left(\prod_{l=0}^{L} H_{p_{l}}\right) \log _{2}\left(1+\sum_{l=0}^{L} 10^{\left(\sqrt{2} \tilde{\sigma}_{Y_{l}} x_{p_{l}}-\tilde{\mu}_{Y_{l}}\right) / 10}\right)\right]
$$

$$
\tilde{m}_{\gamma_{M R C}}^{(q)}=\left(\frac{10}{\ln (10)}\right)^{q} \sum_{p_{0,1}=1}^{N_{p}} \ldots \sum_{p_{N_{0}, 1}=1}^{N_{p}} \cdots \sum_{p_{1, L}=1}^{N_{p}} \ldots \sum_{p_{N_{L}, L}=1}^{N_{p}}\left\{\left[\prod_{l=0}^{L} \Pi_{l}\left(\mathbf{p}_{l}\right)\right]\left[\ln \left(\sum_{l=0}^{L} \frac{1}{\Omega_{l}\left(\mathbf{p}_{l}\right)}\right)\right]^{q}\right\}
$$

$$
\tilde{m}_{\gamma_{M R C}}^{(q)}=\left(\frac{10}{\ln (10)}\right)^{q}\left(\frac{1}{\sqrt{\pi}}\right)^{L+1} \sum_{p_{0}=1}^{N_{p}} \sum_{p_{1}=1}^{N_{p}} \cdots \sum_{p_{L}=1}^{N_{p}}\left\{\left(\prod_{l=0}^{L} H_{p_{l}}\right)\left[\ln \left(\sum_{l=0}^{L} 10^{\left(\sqrt{2} \tilde{\sigma}_{Y_{l}} x_{p}-\tilde{\mu}_{Y_{l}}\right) / 10}\right)\right]^{q}\right\}
$$

relation:

$$
M_{Y}(s)=E\left\{e^{-s Y}\right\}=\frac{1}{\ln (2)} \frac{1}{\Gamma(s)} \int_{0}^{+\infty} \xi^{s-1} e^{-\xi} M_{X}(\xi) d \xi
$$

Proof: Theorem 1 follows from [34, Vol. 4, Eq. (1.1.3.4)] and some algebraic manipulations.

Using Theorem 1, the MGF of $\mathrm{C}\left(\gamma_{M R C} ; M+1\right)$ can be computed by first putting (29) into (31), and then solving the related integral. Then, EC, i.e., the statistical expectation of $C(\cdot ; \cdot)$, can be obtained via simple differentiation [2], i.e, $\mathrm{EC}^{\mathrm{MRC}, \mathrm{I}-\mathrm{SY}}\left(\gamma_{M R C} ; M+1\right) \cong$ $-d M_{C\left(\gamma_{M R C} ; M+1\right)}^{\mathrm{I}-\mathrm{SY}}(s) /\left.d s\right|_{s=0}$, as shown in (32) on top of this page.

2) Maximal Ratio Combining - Method 2: The second method foresees to approximate, in a single step, the endto-end SNR $\gamma_{M R C}$ with a Log-Normal RV, i.e., $\gamma_{M R C} \sim$ $\log N\left(\tilde{\mu}_{\gamma_{M R C}}, \tilde{\sigma}_{\gamma_{M R C}}\right)$, where $\tilde{\mu}_{\gamma_{M R C}}$ and $\tilde{\sigma}_{\gamma_{M R C}}$ are obtained by applying the I-SY method using (15) and Appendix II. Accordingly, we have $\tilde{\mu}_{\gamma_{M R C}}=\tilde{m}_{\gamma_{M R C}}^{(1)}$ and $\tilde{\sigma}_{\gamma_{M R C}}=$ $\sqrt{\tilde{m}_{\gamma_{M R C}}^{(2)}-\left(\tilde{m}_{\gamma_{M R C}}^{(1)}\right)^{2}}$, with $\tilde{m}_{\gamma_{M R C}}^{(q)}$ being defined in (33) shown on top of this page. Finally, ABEP, $\mathrm{P}_{\text {out }}$ and EC are computed from (21)-(23) by setting $\tilde{\mu}_{Y_{l}}=-\tilde{\mu}_{\gamma_{M R C}}$ and $\tilde{\sigma}_{Y_{l}}=\tilde{\sigma}_{\gamma_{M R C}}$.

3) Maximal Ratio Combining - Method 3: Similar to the first approximation method, also the third one foresees two main steps: i) $\gamma_{l}$ is approximated by using the I-SY method, i.e., $\gamma_{l} \sim \log N\left(-\tilde{\mu}_{Y_{l}}, \tilde{\sigma}_{Y_{l}}\right)$, and ii) then, a second $\log -$ Normal approximation is applied to the resulting Log-Normal power-sum, i.e., $\gamma_{M R C} \sim \log N\left(\tilde{\mu}_{\gamma_{M R C}}, \tilde{\sigma}_{\gamma_{M R C}}\right)$. So, while methods 1 and 2 foresee a single Log-Normal approximation, method 3 applies the I-SY method twice.

From the analytical point of view, the performance metrics of method 3 can be derived from the analysis developed for method 2. In particular, while to compute $\tilde{\mu}_{\gamma_{M R C}}$ and $\tilde{\sigma}_{\gamma_{M R C}}$ in (33) we have used the MGF in (15), now these
TABLE I

COMPUTATIONAL COMPLEXITY, O (.), OF THE PROPOSED METHODS FOR ESTIMATING $\mathrm{P}_{\text {out }} Q Q$ AND $N$ ARE PARAMETERS OF THE EULER-SUM BASED FRAMEWORK IN [2].

\begin{tabular}{|c|c|}
\hline Method & Complexity O (.) \\
\hline \hline GQR (SC) & $\sum_{l=0}^{L} N_{p}^{N_{l}}+L+1$ \\
\hline GQR (MRC) & $N_{p}^{\sum_{l=0}^{L} N_{l}}$ \\
\hline I-SY (SC) & $\sum_{l=0}^{L} N_{p}^{N_{l}}+L+1$ \\
\hline I-SY (MRC 1) & $\sum_{l=0}^{L} N_{p}^{N_{l}}+N_{p}^{L+1}+Q N$ \\
\hline I-SY (MRC 2) & $N_{p}^{\sum_{l=0}^{L} N_{l}}$ \\
\hline I-SY (MRC 3) & $\sum_{l=0}^{L} N_{p}^{N_{l}}+N_{p}^{L+1}$ \\
\hline
\end{tabular}

parameters can be obtained from the MGF in (29). After some algebraic manipulations, we have $\tilde{\mu}_{\gamma_{M R C}}=\tilde{m}_{\gamma_{M R C}}^{(1)}$ and $\tilde{\sigma}_{\gamma_{M R C}}=\sqrt{\tilde{m}_{\gamma_{M R C}}^{(2)}-\left(\tilde{m}_{\gamma_{M R C}}^{(1)}\right)^{2}}$, where $\tilde{m}_{\gamma_{M R C}}^{(q)}$ is defined in (34) on top of this page. Finally, ABEP, $\mathrm{P}_{\text {out }}$ and EC can be still obtained using the same formulas as for method 2 .

\section{Computational Complexity of the Proposed METHODS}

In this section, we analyze the computational complexity of all methods proposed for performance analysis of cooperative multi-hop wireless networks over Log-Normal fading environments. The aim is to show that, in general, the ISY method allows to reduce the computational effort for estimating important performance metrics.

In particular, Table I shows an approximate analysis of the computational complexity for estimating $\mathrm{P}_{\text {out }}$ for all system setups analyzed in the manuscript. Similar computational complexities can be obtained for other performance metrics. 
We emphasize that to get insightful information about the computational effort required by each method, the results in Table I have to be analyzed carefully. In what follows, we will provide some guidelines to interpret them correctly.

Let us start by comparing the complexity of the GQRbased and I-SY methods when SC diversity is considered. From Table I, we can observe that both approaches seem to have the same computational complexity in terms of number of points $N_{p}$. However, this result must be analyzed with further attention. As a matter of fact, in general, the number of points $N_{p}$ to get the desired accuracy is significantly different for GQR-based and I-SY methods. As mentioned in Section III, using the GQR-based method to compute $\mathrm{P}_{\text {out }}$ (as well as other performance metrics) may require a very large number of points (e.g., > 100), as it will be shown in Section VI. On the contrary, the I-SY approximation just foresees to compute the log-moments of the power-sum of correlated Log-Normal RVs, which needs a number of points $5<N_{p}<10$ [27] to get good estimates (see Section VI). In conclusion, when comparing, in Table I, GQR-based and I-SY methods we should also take into consideration that $N_{p}^{G Q R} \gg N_{p}^{I-S Y}$ when computing $\mathrm{P}_{\text {out }}$. A similar comment holds when comparing the performance of MRC diversity (in particular for method 2).

Let us now compare GQR-based and I-SY methods when MRC diversity is considered. We observe that the simplest approximation is method 3 , while the most complicated is method 2. Similar to the SC case, the three I-SY approximation methods are, in general, much simpler to be computed than GQR-based method. We can also observe that methods 1 and 3 have similar computational complexities. However, numerical results will indicate (see Section VI) that the first one is more accurate as only one Log-Normal approximation is needed. However, method 3 is the only one that can be readily generalized to reference scenarios with correlated diversity branches.

\section{NumericAl AND Simulation Results}

In this section, we provide some numerical and simulation results to validate the accuracy of the proposed frameworks. In particular, the following system setup is considered: i) we assume that every relay channel is Log-Normal distributed with mean $\mu=\mu_{l, n}=0 \mathrm{~dB}$, and standard deviation uniformly and independently (for every hop) distributed within the range $[3,9] \mathrm{dB}^{6}$, ii) independent fading is assumed among the branches and hops, iii) for blind relays we assume $G=G_{l, n}=1$, iv) for the sake of simplicity, the direct link is assumed to be very week in the simulation scenario and, without loss of generality, is neglected from the analysis, and

\footnotetext{
${ }^{6}$ While the accuracy of the framework in Section III is almost insensitive to the parameters of Log-Normal RVs, the accuracy of the I-SY method may depend on these parameters. Although it is very hard to analyze the accuracy of an approximation for every system parameter setup, we consider here one of worst reference scenarios for Log-Normal approximation. In fact, the reference scenario composed by Log-Normal summands with zero mean and different $\mathrm{dB}$ spreads leads to the worst accuracy when a Log-Normal $\mathrm{RV}$ is used to approximate the Log-Normal power-sum [38]. We have also verified by simulation that the accuracy of the I-SY method improves when either the $\mathrm{dB}$ spreads are the same or when the $\mathrm{dB}$ spreads are smaller than $3 \mathrm{~dB}$.
}

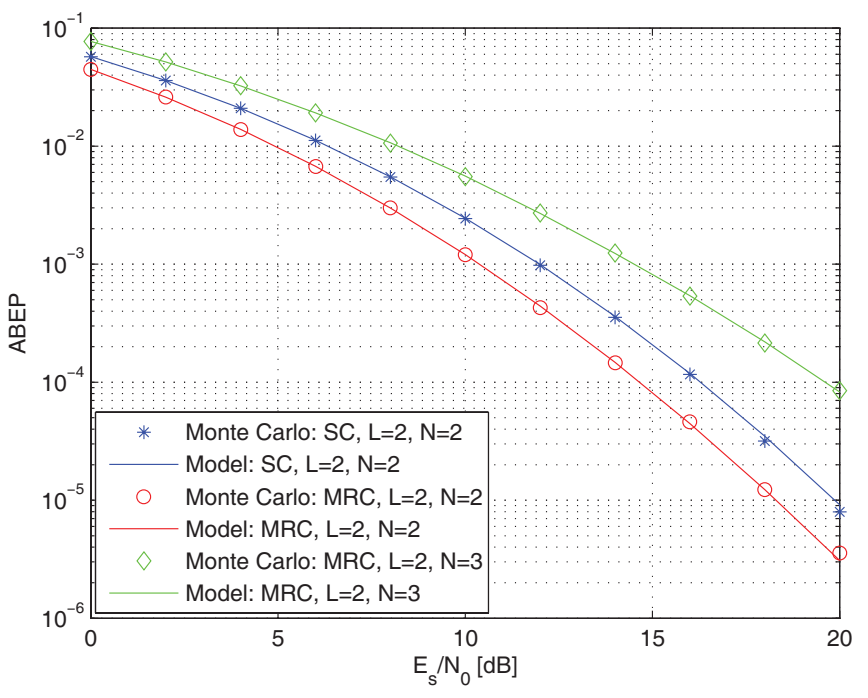

Fig. 1. Average Bit Error Probability (framework in Section III) for blind relays (Binary Phase Shift Keying modulation, i.e., $b=1, a=2$ ). Comparison of analysis $\left(N_{p}=10\right.$ for MRC and $N_{p}=200$ for SC) and Monte Carlo simulation $\left(N=N_{l} \forall l\right)$.

v) the number of GQR points $N_{p}$ is chosen according to the guidelines given in Section V (see the captions of the figures for specific values), and Monte Carlo simulation results are obtained by averaging over $10^{6}$ points.

In Figs. 1-3, we compare Monte Carlo simulation and GQR-based approximation for blind relays. We observe that the proposed method almost overlaps with Monte Carlo simulations, thus substantiating the correctness and accuracy of the developed formulas. In Figs. 4-8, we analyze the accuracy of the I-SY approximation for blind relays as well. In particular, SC diversity is analyzed in Figs. 4, 5. We observe that reasonably accurate estimates of the ABEP can be obtained, and the approximation does not introduce error floors for high $E_{s} / \mathscr{N}_{0}$ values. We also observe that very accurate results are obtained when estimating EC. This is a general result: the proposed I-SY framework can predict EC in a very accurate way, and, in general, the number of points $N_{p}$ required for approximating it is small (less than 10). We also note that, in general, the approximation accuracy gets worse as $L$ increases, while it does not change significantly with $N_{l}$. This is because a Log-Normal approximation is used in every diversity branch and, so, approximation errors may accumulate for large $L$. However, the overall error is less than $1.5 \mathrm{~dB}$ for very small values of ABEP. In Figs. 6-8, we analyze the accuracy of ISY method for MRC diversity. We observe that methods 1 and 2 provide almost the same accuracy, with the former method being simpler than the latter to be computed. Also method 3 , the simplest one to be computed, offers a good accuracy without error floors for high $E_{s} / \mathscr{N}_{0}$, but, as expected, since the Log-Normal approximation is applied twice, it provides less accurate estimates, even though the error is, in general, tolerable. From this analysis, we can conclude that method 1 provides the required trade-off between accuracy and computational simplicity to be used as a fast, simple, yet accurate approach for performance prediction of cooperative multi-hop networks over Log-Normal channels and MRC diversity.

Furthermore, we observe, e.g., in Fig. 4 and Figs. 6-8, that 


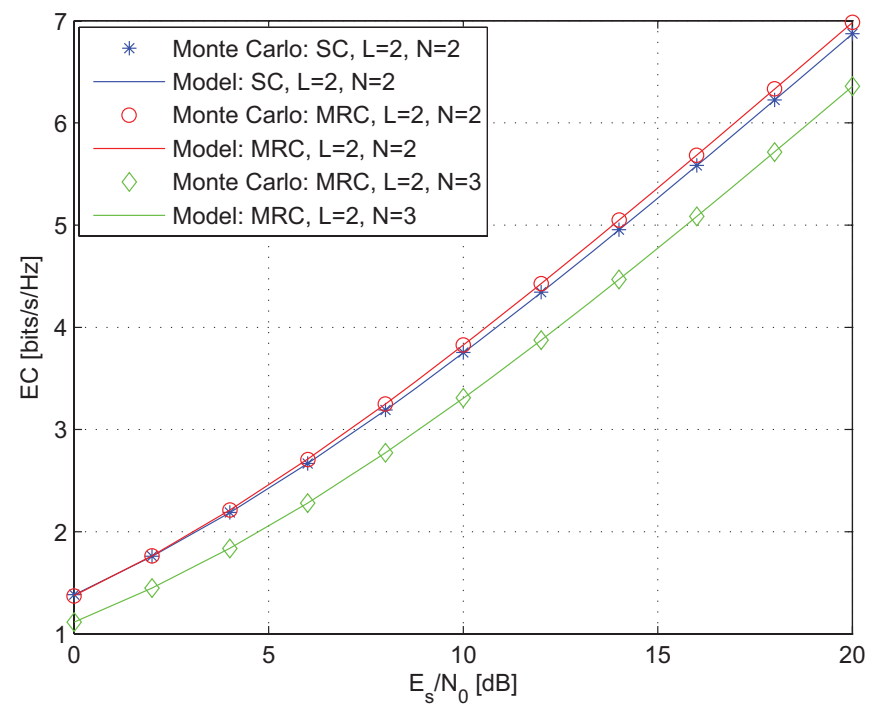

Fig. 2. Ergodic (Shannon) Capacity (framework in Section III) for blind relays. Comparison of analysis $\left(N_{p}=10\right.$ for MRC and $N_{p}=200$ for SC) and Monte Carlo simulation ( $N=N_{l} \forall l$ ). Note that, for ease of illustration, the Ergodic Capacity shown in the figure is multiplied by the scaling factor $M+1$, which accounts for the number of time slots required by the repetitionbased cooperative diversity scheme assumed in this paper.

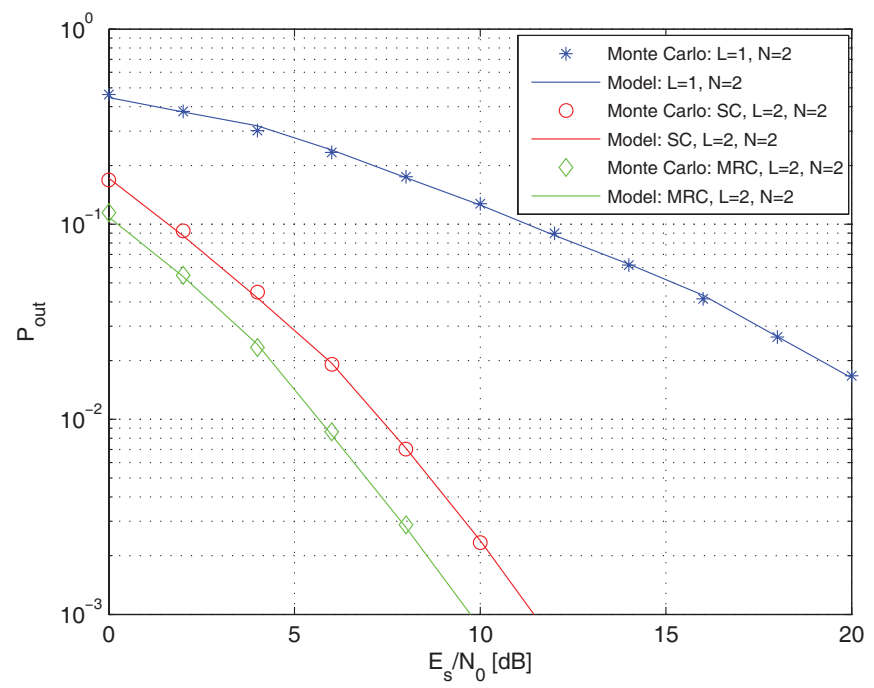

Fig. 3. Outage Probability (framework in Section III) for blind relays $\left(V_{T}=\right.$ $-5 \mathrm{~dB})$. Comparison of analysis $\left(N_{p}=200\right)$ and Monte Carlo simulation $\left(N=N_{l} \forall l\right)$.

the accuracy of our approximations gets, in general, worse in the high-SNR region. Our empirical trials have shown that this effect is mainly due to the used Log-Normal approximation rather than to the number of points $N_{p}$ required to compute the log-moments needed for the approximation. As a matter of fact, the number of points used for these numerical results is either $N_{p}=10$ or $N_{p}=5$ (only in Fig. 7 for $L=3$ and $N=3$ for reducing the computational complexity), which, as shown in [27], provide, in general, almost exact estimates. This conclusion is also substantiated by the fact that the computation of EC does not show this problem.

In Figs. 9-11, we analyze the accuracy of the frameworks when CSI-assisted relays are considered. In particular, in Fig. 9 we study the accuracy of GQR-based method (see

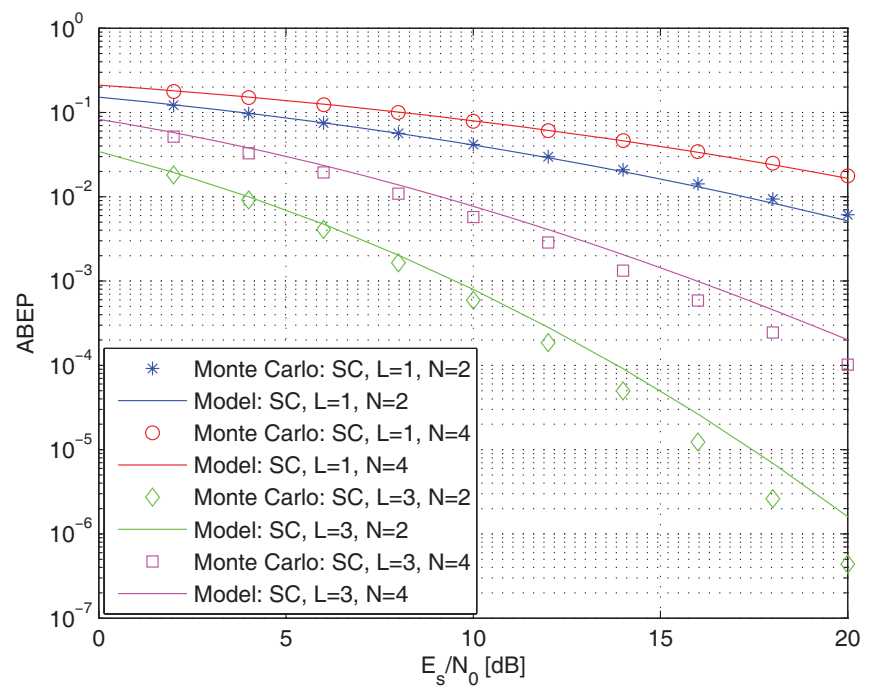

Fig. 4. Average Bit Error Probability (framework in Section IV-B) for blind relays (Binary Phase Shift Keying modulation, i.e., $b=1, a=2$ ). Comparison of analysis $\left(N_{p}=10\right.$ in (20)) and Monte Carlo simulation $\left(N=N_{l} \forall l\right)$

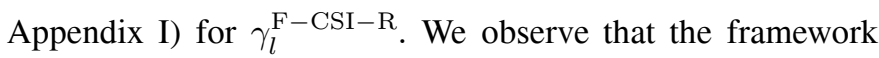
is very accurate, and the results also show that the simpler formulas developed for $\gamma_{l}^{\mathrm{F}-\mathrm{CSI}-\mathrm{I}}$ provide a tight lower bound for performance prediction (ABEP) of $\gamma_{l}^{\mathrm{F}-\mathrm{CSI}-\mathrm{R}}$ as well. Furthermore, in Figs. 10, 11 we study the accuracy of the ISY method when used to estimate EC and ABEP, respectively $\left(\gamma_{l}^{\mathrm{F}-\mathrm{CSI}-\mathrm{I}}\right.$ system setup). We observe that also in this case a good accuracy is retained, and the framework can be used for performance analysis of this scenario as well. In Fig. 12 we investigate the accuracy of I-SY method for semi-blind relays. In particular, simulated and analytical $\mathrm{P}_{\text {out }}$ are compared for SC diversity and several system setups. We observe a good overlapping between model and simulation.

Finally, in Fig. 13 we analyze the accuracy of the proposed I-SY approximation for a large number of hops and branches. The figure shows the results when the F-CSI-I system setup is considered, and for cooperative networks formed by up to 90 relays (i.e., $L=10$ and $N=N_{l}=10 \forall l$ ). We can see a very good overlapping between the curves. In particular, with the aim to show that the fading parameters chosen in the previous figures are very challenging from the approximation point of view, we have considered smaller values of the fading standard deviation. We can observe that in this case a good approximation is retained for a very small number of GQR points, i.e., $N_{p}=3$, which results in a very simple I-SY analytical framework for performance analysis and design.

In summary, the obtained results confirm that the proposed framework is general enough and simple for a sound system design and optimization of cooperative systems over LogNormal fading channels, and for various system settings and relay strategies. Depending on the designer's requirements, the appropriate method for performance prediction may be used to meet the desired targets of accuracy, simplicity, and flexibility. In particular, both computational complexity analysis in Section V and numerical results in Section VI lead to the following conclusions when comparing GQR-based and I-SY methods: in general, i) when SC diversity is considered 


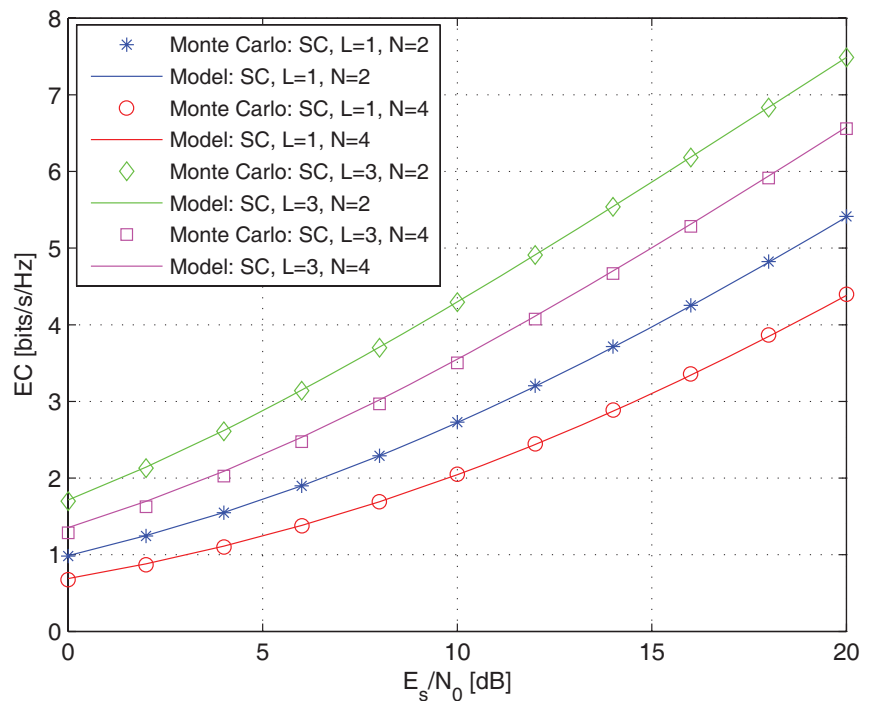

Fig. 5. Ergodic (Shannon) Capacity (framework in Section IV-B) for blind relays. Comparison of analysis $\left(N_{p}=10\right.$ in (20)) and Monte Carlo simulation $\left(N=N_{l} \forall l\right)$. Note that, for ease of illustration, the Ergodic Capacity shown in the figure is multiplied by the scaling factor $M+1$, which accounts for the number of time slots required by the repetition-based cooperative diversity scheme assumed in this paper.

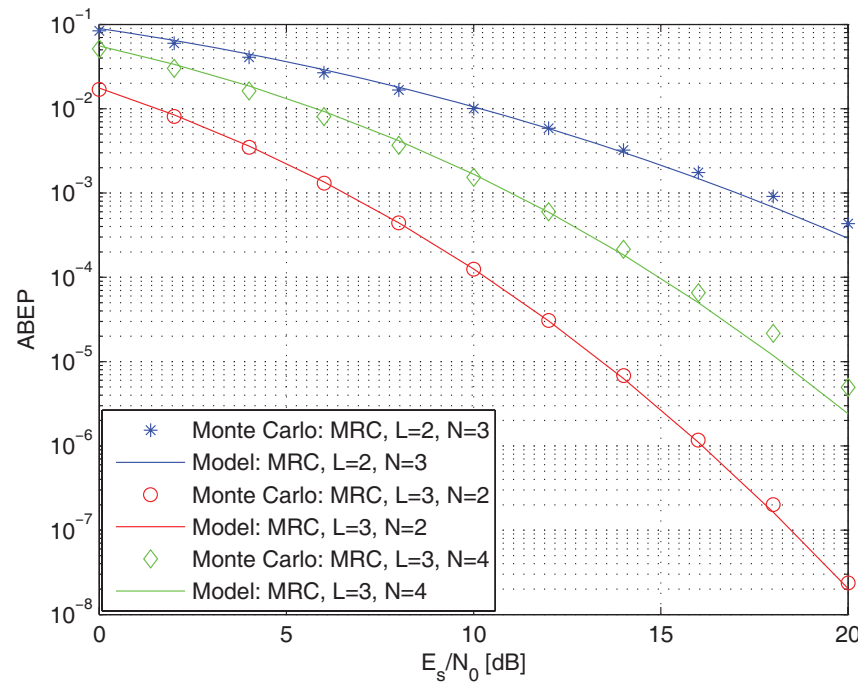

Fig. 6. Average Bit Error Probability (framework in Section IV-C, Method 1) for blind relays (Binary Phase Shift Keying modulation, i.e., $b=1, a=2$ ). Comparison of analysis $\left(N_{p}=10\right.$ in (20)) and Monte Carlo simulation $\left(N=N_{l} \forall l\right)$.

the I-SY framework allows to reduce the number of points $N_{p}$ required to compute all performance metrics of interest, while keeping the same number of fold summations; and ii) when MRC diversity is considered the I-SY framework (i.e., method 1) allows to reduce the number of fold summations required to compute all performance metrics of interest, while keeping the same number of $N_{p}$ points.

\section{CONCLUSIONS}

In this paper, we have developed a comprehensive framework for computing the performance of cooperative multi-hop systems over Log-Normal fading channels. In particular, two main frameworks have been proposed. The first one, which

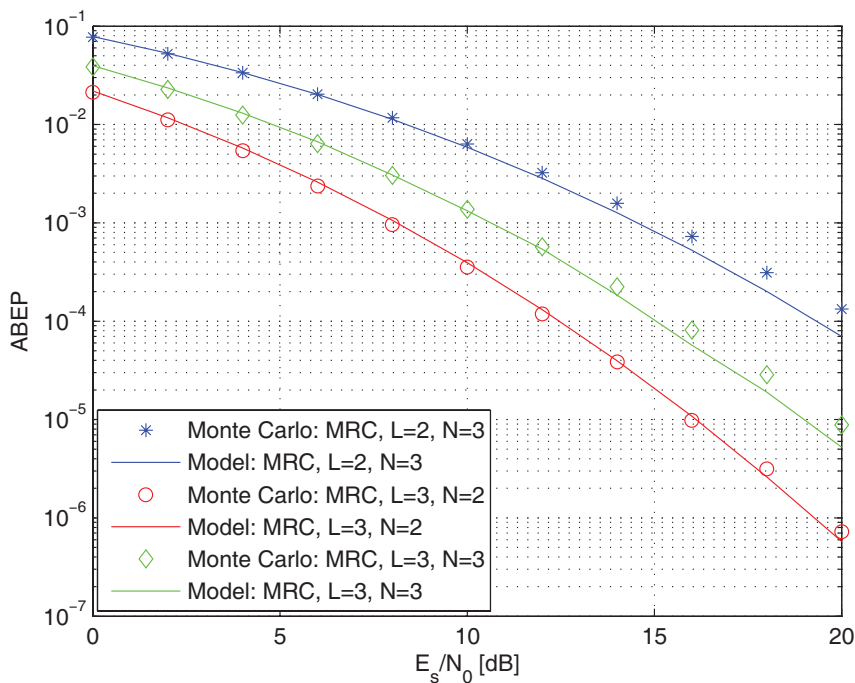

Fig. 7. Average Bit Error Probability (framework in Section IV-C, Method 2) for blind relays (Binary Phase Shift Keying modulation, i.e., $b=1, a=2$ ). Comparison of analysis $\left(N_{p}=10\right.$ in (20), and $N_{p}=5$ in (20) for $L=3$ and $N=3$ for reducing the computational complexity) and Monte Carlo simulation $\left(N=N_{l} \forall l\right)$

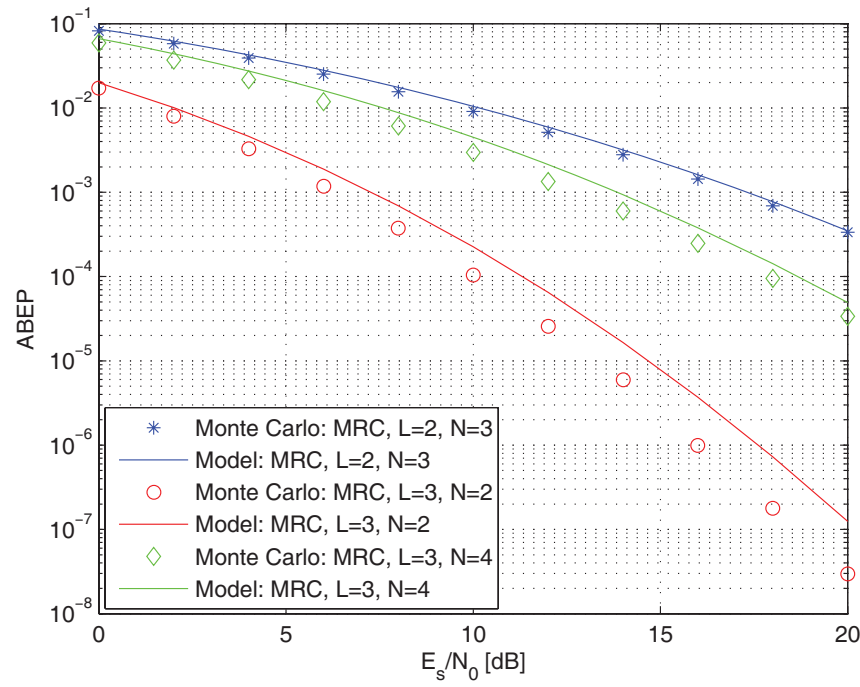

Fig. 8. Average Bit Error Probability (framework in Section IV-C, Method 3) for blind relays (Binary Phase Shift Keying modulation, i.e., $b=1, a=2$ ). Comparison of analysis $\left(N_{p}=10\right.$ in (20)) and Monte Carlo simulation $\left(N=N_{l} \forall l\right)$

is based on the accurate GQR representation of the MGF of a Log-Normal RV, may provide very accurate estimates for important performance indexes, but its computational complexity may become high when both the number of hops and diversity branches get large. The second one, which is based on the I-SY approximation technique, provides, in general, less accurate results than the first framework, but with a significant reduction in computational complexity. In summary, the results confirm that the I-SY method can be efficiently used for performance analysis and system design of cooperative multi-hop wireless systems in the vast majority of system setups of practical interest, and may be exploited for further analysis and system optimization of upper layers of the protocol stack. On the other hand, when either the number of 


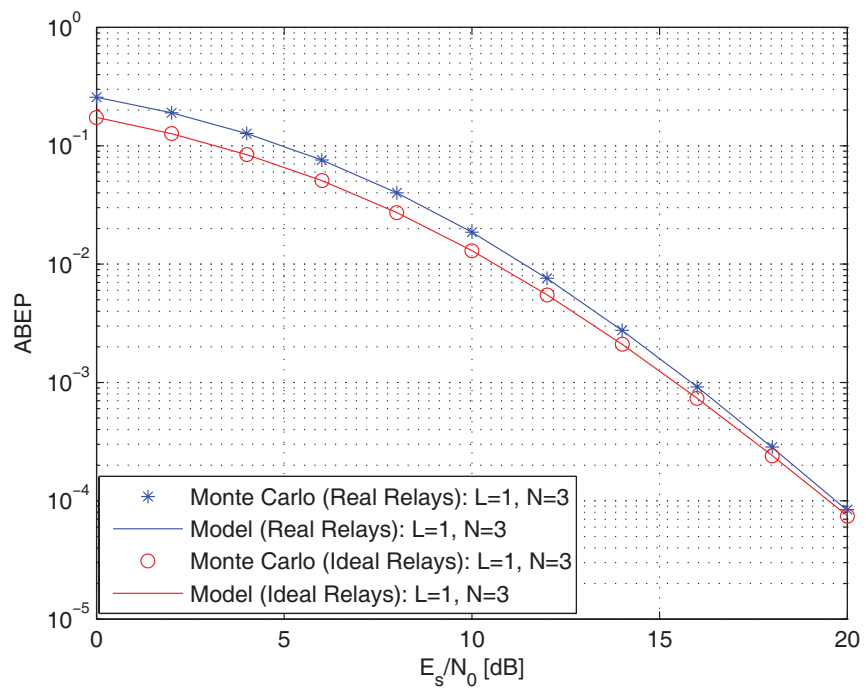

Fig. 9. Average Bit Error Probability (framework in Section III and Appendix

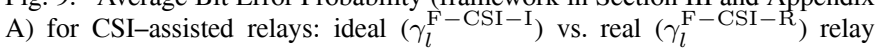
gains (Binary Phase Shift Keying modulation, i.e., $b=1, a=2$ ). Comparison of analysis $\left(N_{p}=30\right)$ and Monte Carlo simulation $\left(N=N_{l} \forall l\right)$.

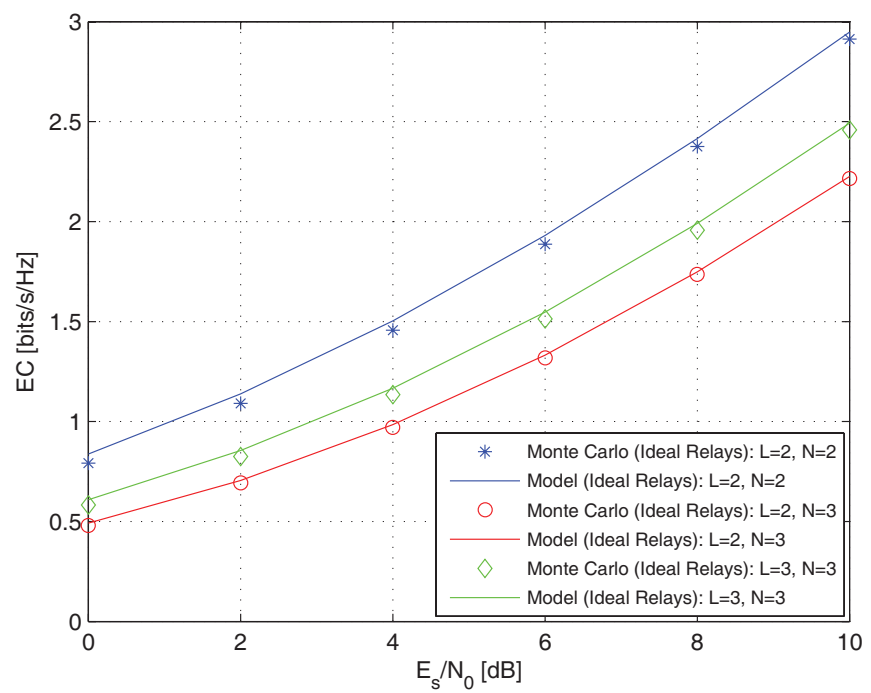

Fig. 10. Ergodic (Shannon) Capacity (framework in Section IV, Selection Combining) for CSI-assisted relays $\left(\gamma_{l}^{\mathrm{F}-\mathrm{CSI}-\mathrm{I}}\right.$ system setup). Comparison of analysis $\left(N_{p}=10\right.$ in (20)) and Monte Carlo simulation $\left(N=N_{l} \forall l\right)$. Note that, for ease of illustration, the Ergodic Capacity shown in the figure is multiplied by the scaling factor $M+1$, which accounts for the number of time slots required by the repetition-based cooperative diversity scheme assumed in this paper.

hops or diversity branches is small, the GQR-based method represents a very accurate yet flexible tool for system analysis and optimization.

\section{APPENDIX A}

\section{CASE STUdy FOR THE ANALYSIS OF $\gamma_{l}^{\mathrm{F}-\mathrm{CSI}-\mathrm{R}}$}

The aim of this Appendix is to show that even the complicated SNR $\gamma_{l}^{\mathrm{F}-\mathrm{CSI}-\mathrm{R}}$ defined in Section II can be easily written as the power-sum of generically correlated LogNormal RVs.

First of all, let us re-write $\gamma_{l}^{\mathrm{F}-\mathrm{CSI}-\mathrm{R}}$ in an alternative form, as shown in (35) on top of the next page. Then, by relying on the closure property of Log-Normal RVs, it can be easily

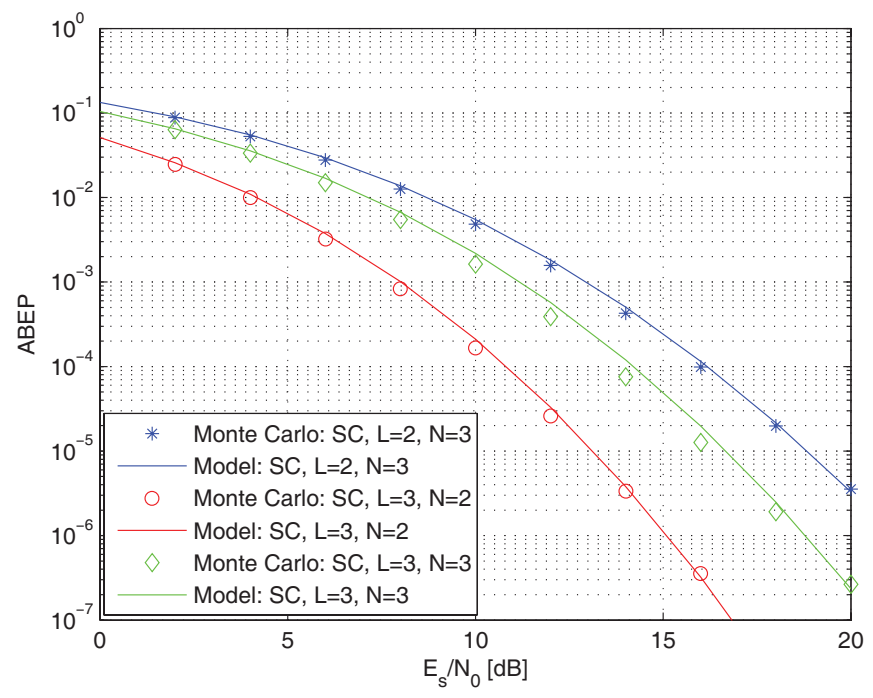

Fig. 11. Average Bit Error Probability (framework in Section IV, Selection Combining) for CSI-assisted relays $\left(\gamma_{l}^{\mathrm{F}-\mathrm{CSI}-\mathrm{I}}\right.$ system setup). Comparison of analysis $\left(N_{p}=10\right.$ in (20)) and Monte Carlo simulation $\left(N=N_{l} \forall l\right)$.

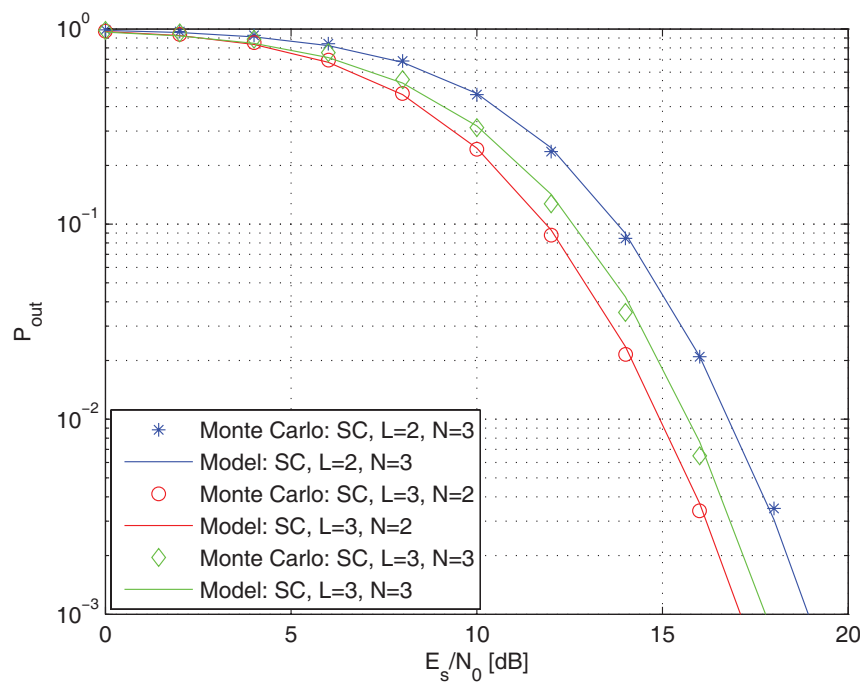

Fig. 12. Outage Probability (framework in Section IV, Selection Combining) for semi-blind relays $\left(V_{T}=10 \mathrm{~dB}\right)$. Comparison of analysis $\left(N_{p}=10 \mathrm{in}\right.$ (20)) and Monte Carlo simulation $\left(N=N_{l} \forall l\right)$.

argued from (35) that $\gamma_{l}^{\mathrm{F}-\mathrm{CSI}-\mathrm{R}}$ is given by the power-sum of correlated Log-Normal RVs. For the sake of simplicity, let us consider, as an example, the scenario with $N_{l}=3$. The interested reader may use the same development for whatever number of hops. In such a case, (35) simplifies as follows (subscript $l$ is neglected for simplicity):

$$
\begin{aligned}
\left(\gamma_{l}^{\mathrm{F}-\mathrm{CSI}-\mathrm{R}}\right)^{-1} & =\gamma_{1}^{-1}+\gamma_{2}^{-1}+\gamma_{3}^{-1}+\gamma_{1}^{-1} \gamma_{2}^{-1} \\
& +\gamma_{1}^{-1} \gamma_{3}^{-1}+\gamma_{2}^{-1} \gamma_{3}^{-1}+\gamma_{1}^{-1} \gamma_{2}^{-1} \gamma_{3}^{-1}
\end{aligned}
$$

The SNR in (36) is given by the summation of 7 correlated Log-Normal RVs, i.e., $Y=\sum_{n=1}^{7} Y_{n}$ with $\left\{Y_{n}\right\}_{n=1}^{7}$ given in 


$$
\begin{aligned}
\left(\gamma_{l}^{\mathrm{P}-\mathrm{CSI}-\mathrm{R}}\right)^{-1} & =(1 !)^{-1} \sum_{n=1}^{N_{l}}\left(\gamma_{l, n}^{-1}\right)+(2 !)^{-1} \sum_{n_{1}=1}^{N_{l}} \sum_{n_{2} \neq n_{1}=1}^{N_{l}}\left(\gamma_{l, n_{1}}^{-1} \cdot \gamma_{l, n_{2}}^{-1}\right) \\
& +\cdots+\left(N_{l} !\right)^{-1} \sum_{n_{1}=1}^{N_{l}} \sum_{n_{2} \neq n_{1}=1}^{N_{l}} \ldots \sum_{n_{N_{l}} \neq n_{N_{l}-1} \neq \ldots \neq n_{1}=1}^{N_{l}}\left(\gamma_{l, n_{1}}^{-1} \cdot \gamma_{l, n_{2}}^{-1} \cdot \ldots \cdot \gamma_{l, n_{N_{l}}}^{-1}\right)
\end{aligned}
$$

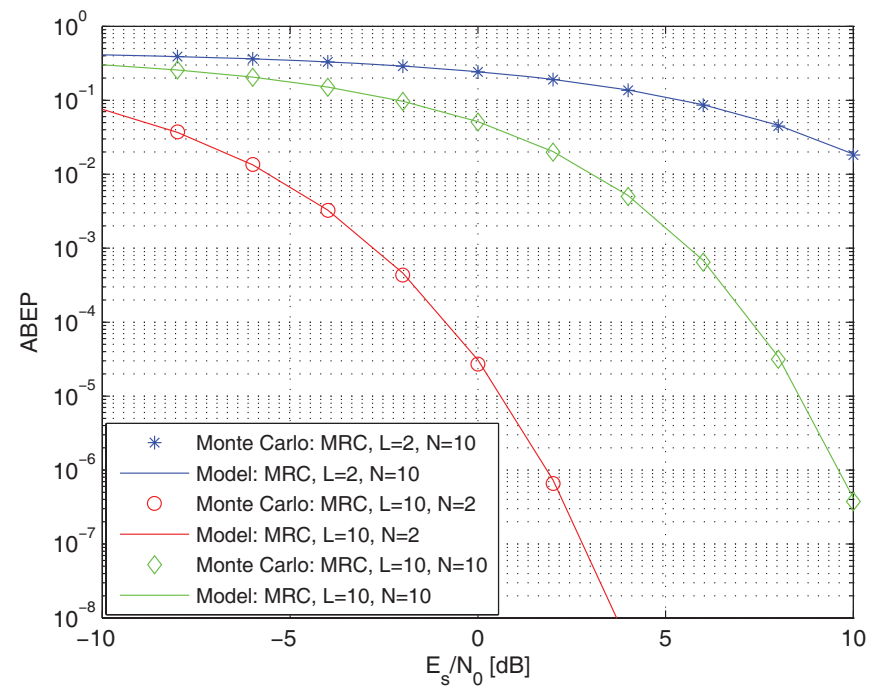

Fig. 13. Average Bit Error Probability (framework in Section IV-C, Method 1) for CSI-assisted relays ( $\gamma_{l}^{\mathrm{F}-\mathrm{CSI}-\mathrm{I}}$ system setup). Comparison of analysis $\left(N_{p}=3\right.$ in (20)) and Monte Carlo simulation $\left(N=N_{l} \forall l\right)$. Setup: $\mu=\mu_{l, n}=0 \mathrm{~dB}$, and $\sigma_{l, n}$ uniformly and independently (for every hop) distributed within the range $[3,6] \mathrm{dB}$.

(37) and $\phi=10 \log _{10}\left(E_{s} / \mathscr{N}_{0}\right)$.

$$
\left\{\begin{array}{l}
Y_{1}=\left(\gamma_{1}\right)^{-1} \sim \log N\left(-\mu_{1}-\phi, \sigma_{1}\right) \\
Y_{2}=\left(\gamma_{2}\right)^{-1} \sim \log N\left(-\mu_{2}-\phi, \sigma_{2}\right) \\
Y_{3}=\left(\gamma_{3}\right)^{-1} \sim \log N\left(-\mu_{3}-\phi, \sigma_{3}\right) \\
Y_{4}=\left(\gamma_{1} \gamma_{2}\right)^{-1} \sim \log N\left(-\mu_{1}-\mu_{2}-2 \phi, \sqrt{\sigma_{1}^{2}+\sigma_{2}^{2}}\right) \\
Y_{5}=\left(\gamma_{1} \gamma_{3}\right)^{-1} \sim \log N\left(-\mu_{1}-\mu_{3}-2 \phi, \sqrt{\sigma_{1}^{2}+\sigma_{3}^{2}}\right) \\
Y_{6}=\left(\gamma_{2} \gamma_{3}\right)^{-1} \sim \log N\left(-\mu_{2}-\mu_{3}-2 \phi, \sqrt{\sigma_{2}^{2}+\sigma_{3}^{2}}\right) \\
Y_{7}=\left(\gamma_{1} \gamma_{2} \gamma_{3}\right)^{-1} \\
\sim \log N\left(-\mu_{1}-\mu_{2}-\mu_{3}-3 \phi, \sqrt{\sigma_{1}^{2}+\sigma_{2}^{2}+\sigma_{3}^{2}}\right)
\end{array}\right.
$$

Accordingly, to use the frameworks developed in Sections III and IV, we need to just compute the mean vector $\left(\boldsymbol{\mu}_{Y}\right)$, and the covariance matrix $\left(\boldsymbol{\Sigma}_{Y}\right)$ of the set of RVs $\left\{Y_{n}\right\}_{n=1}^{7}$. These parameters can be readily computed from [1]. For the sake of conciseness, they are not reported in the present manuscript.

In conclusion, also for this system setup similar frameworks to those developed in Sections III and IV can be developed and accurate performance metrics for $\gamma_{l}^{\mathrm{F}-\mathrm{CSI}-\mathrm{R}}$ can be computed as well.

\section{APPENDIX B \\ I-SY METHOD FOR CORRELATED LOG-NORMAL RVS}

The I-SY method is, in principle, similar to the SY method [37], but the parameters of the approximation are computed without resorting to recursive approximations. In particular, the I-SY method foresees to approximate the power-sum of generically correlated Log-Normal RVs with another LogNormal RV, whose parameters can be obtained from the logarithmic moments of the power-sum [27]. Let us use, for illustrative purposes, the following notation: i) $S$ is a generic power-sum of correlated Log-Normal RVs, ii) $S_{\mathrm{dB}}=$ $10 \log _{10}(S)$, and iii) $\tilde{m}_{S}^{(q)}$ is the $q$-th non-central log-moment of RV $S$, i.e., $\tilde{m}_{S}^{(q)}=E\left\{S_{\mathrm{dB}}^{q}\right\}=E\left\{\left[10 \log _{10}(S)\right]^{q}\right\}$.

The I-SY method works as follows:

- RV $S$ is approximated by a Log-Normal RV, i.e., $S \sim$ $\log N\left(\tilde{\mu}_{S}, \tilde{\sigma}_{S}\right)$, where $\tilde{\mu}_{S}$ and $\tilde{\sigma}_{S}$ are the parameters (in $\mathrm{dB})$ of the approximating PDF.

- $\tilde{\mu}_{S}$ and $\tilde{\sigma}_{S}$ are computed from $\tilde{m}_{S}^{(q)}$ as follows: $\tilde{\mu}_{S}=$

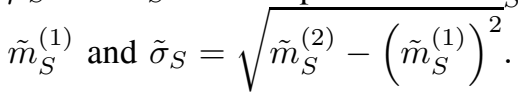

So, the I-SY approximation method boils down to the computation of $\tilde{m}_{S}^{(q)}$. This is done in two steps:

1) First, the MGF, $M_{S_{\mathrm{dB}}}(\cdot)$, of RV $S_{\mathrm{dB}}$ is computed from the MGF of $S$ as follows [27, Eq. (6)]: $M_{S_{\mathrm{dB}}}(p)=$ $[\Gamma(p)]^{-1} \int_{0}^{+\infty} z^{p-1} M_{S}(z) d z$.

2) Second, the log-moments are computed via differentiation: $\tilde{m}_{S}^{(q)}=(-1)^{q} d^{q} M_{S_{\mathrm{dB}}}(p) /\left.d p^{q}\right|_{p=0}$.

An explicit closed-form expression of $\tilde{m}_{S}^{(q)}$ requires the MGF of $S$ to be known in closed-form. Such an expression can be found in [32, Eq. (17)] using GQR integration. So, using [32], and steps 1 and 2 above, as well as simple algebraic manipulations described in [27], the log-moments $\tilde{m}_{S}^{(q)}$ can be readily written as shown in (20).

\section{REFERENCES}

[1] G. L. Stüber, Principles of Mobile Communications, 2nd ed. Kluwer Academic Publisher, 2001.

[2] M. K. Simon and M.-S. Alouini, Digital Communication over Fading Channels, 1st ed. John Wiley \& Sons, 2000.

[3] G. J. Foschini and M. J. Gans, "On limits of wireless communications in a fading environment when using multiple antennas," Wireless Personal Commun., vol. 6, no. 3, pp. 311-335, 1998.

[4] A. Sendonaris, E. Erkip, and B. Aazhang, "User cooperation diversitypart I: system description; part II: implementation aspects and performance analysis," IEEE Trans. Commun., vol. 51, no. 11, pp. 1927-1948, Nov. 2003.

[5] J. Boyer, D. D. Falconer, and H. Yanikomeroglu, "Multihop diversity in wireless relaying channels," IEEE Trans. Commun., vol. 52, no. 10, pp. 1820-1830, Oct. 2004.

[6] J. Laneman, D. Tse, and G. Wornell, "Cooperative diversity in wireless networks: efficient protocols and outage behavior," IEEE Trans. Inf. Theory, vol. 50, no. 12, pp. 3062-3080, Dec. 2004. 
[7] M. O. Hasna and M.-S. Alouini, "Outage probability of multihop transmission over Nakagami fading channels," IEEE Commun. Lett., vol. 7, no. 5, pp. 216-218, May 2003.

[8] M. O. Hasna and M.-S. Alouini, "Harmonic mean and end-to-end performance of transmission systems with relays," IEEE Trans. Commun., vol. 52, no. 1, pp. 130-135, Jan. 2004.

[9] P. A. Anghel and M. Kaveh, "Exact symbol error probability of a cooperative network in a Rayleigh-fading environment," IEEE Trans. Wireless Commun., vol. 3, no. 5, pp. 1416-1421, Sep. 2004.

[10] M. O. Hasna and M.-S. Alouini, "A performance study of dual-hop tranmission with fixed gain relays," IEEE Trans. Wireless Commun., vol. 3, no. 6, pp. 1963-1968, Nov. 2004.

[11] G. K. Karagiannidis, T. A. Tsiftsis, and R. K. Mallik, "Bounds for multihop relayed communications in Nakagami- $m$ fading," IEEE Trans. Commun., vol. 54, no. 1, pp. 18-22, Jan. 2006.

[12] G. K. Karagiannidis, "Performance bounds of multihop wireless communications with blind relays over generalized fading channels," IEEE Trans. Wireless Commun., vol. 5, no. 3, pp. 498-503, Mar. 2006.

[13] S. Ikki and M. H. Ahmed, "Performance of multi-hop relaying systems over Weibull fading channels," New Technol., Mobility Security, Springer, pp. 31-38, Nov. 2007.

[14] M. O. Hasna and M.-S. Alouini, "End-to-end outage probability of multihop transmission over lognormal shadowing channels," Arabian J. Science Engineering, vol. 28, no. 2C, pp. 35-44, Dec. 2003.

[15] M. Safari, M. Uysal, "Cooperative diversity over log-normal fading channels: performance analysis and optimization," IEEE Trans. Wireless Commun., vol. 7, no. 5, pp. 1963-1972, May 2008.

[16] M. Di Renzo, F. Graziosi, and F. Santucci, "On the performance of CSIassisted cooperative communications over generalized fading channels," in Proc. IEEE Int. Conf. Commun., May 2008, vol. 1, pp. 1001-1007.

[17] M.-S. Alouini and M. K. Simon, "Dual diversity over correlated lognormal fading channels," IEEE Trans. Commun., vol. 50, no. 12, pp. 1946-1959, Dec. 2002.

[18] M. Pratesi, F. Santucci, and F. Graziosi, "Generalized moment matching for the linear combination of lognormal RVs: application to outage analysis in wireless systems," IEEE Trans. Wireless Commun., vol. 5, no. 5, pp. 1122-1132, May 2006.

[19] R. Ganesh and K. Pahlavan, "Statistical modeling and computer simulation of indoor radio channel," IEE Proc. I, Commun, Speech, Vision, vol. 138, no. 3, pp. 153-161, June 1991.

[20] H. Hashemi, "Impulse response modeling of indoor radio propagation channels," IEEE J. Sel. Areas Commun., vol. 11, no. 7, pp. 967-978, Sep. 1993.

[21] J. Sydir, et al., "Harmonized contribution on 802.16j (mobile multihop relay) usage models," IEEE 802.16 Broadband Wireless Access Working Group, IEEE 802.16j-06/015, Sep. 2006.

[22] K. Pawlikowski, H.-D. Jeong, and J.-S. Lee, "On credibility of simulation studies of telecommunication networks," IEEE Commun. Mag., vol. 40, no. 1, pp. 132-139, Jan. 2002.

[23] C. Newport, D. Kotz, Y. Yuan, R. S. Gray, J. Liu, and C. Elliott, "Experimental evaluation of wireless simulation assumption," Simulation, vol. 83, no. 8, pp. 643-661, Sep. 2007.

[24] C. Bettstetter and C. Hartmann, "Connectivity of wireless multihop networks in a shadow fading environment," ACM/Springer Wireless Networks, vol. 11, no. 5, pp. 571-579, Sep. 2005.

[25] N. Patwari and P. Agrawal, "Effects of correlated shadowing: connectivity, localization, and RF tomography," in Proc. IEEE/ACM Int. Conf. Inform. Processing Sensor Networks, Apr. 2008, vol. 1, pp. 82-93.

[26] P. Agrawal and N. Patwari, "Correlated link shadow fading in multi-hop wireless networks," IEEE Trans. Wireless Commun., vol. 8, no. 8, pp. 4024-4036, Aug. 2009.

[27] M. Di Renzo, F. Graziosi, and F. Santucci, "A general formula for log-MGF computation: application to the approximation of log-normal power sum via Pearson type IV distribution," in Proc. IEEE Veh. Technol. Conf., May 2008, vol. 1, pp. 999-1003.

[28] A. Laourine, A. Stephenne, and S. Affes, "Estimating the ergodic capacity of log-normal channels," IEEE Commun. Lett., vol. 11, no. 7, pp. 568-570, July 2007.

[29] M. Abramowitz and I. A. Stegun, Handbook of Mathematical Functions with Formulas, Graphs, and Mathematical Tables, 9th ed. New York: Dover, 1972.

[30] N. Cressie, A. S. Davis, J. L. Folks, and G. E. Policello II, "The momentgenerating function and negative integer moments," The American Statistician, vol. 35, no. 3, pp. 148-150, Aug. 1981.

[31] G. H. Golub and J. H. Welsch, "Calculation of Gauss quadrature rules," Math. Comput., vol. 23, no. 106, pp. 221-230, Apr. 1969.
[32] N. B. Mehta, J. Wu, A. F. Molisch, and J. Zhang, "Approximating a sum of random variables with a lognormal," IEEE Trans. Wireless Commun., vol. 6, no. 7, pp. 2690-2699, July 2007.

[33] I. S. Gradshteyn and I. M. Ryzhik, Table of Integrals, Series, and Products, 5th ed. Academic Press, Jan. 1994.

[34] A. P. Prudnikov, Y. A. Brychkov, and O. I. Marichev, Integrals and Series. Vol. 4: Direct Laplace Transforms. Vol. 5: Inverse Laplace Transforms. CRC Pub., Apr. 1992.

[35] D. S. Michalopoulos and G. K. Karagiannidis, "Spectral efficient cooperative communications via spatial signal separation," in Proc. IEEE Int. Conf. Commun., May 2008, vol. 1, pp. 1039-1043.

[36] G. Farhadi and N. C. Beaulieu, "Ergodic capacity analysis of wireless relaying systems in Rayleigh fading," in Proc. IEEE Int. Conf. Commun., May 2008, vol. 1, pp. 3730-3735.

[37] S. C. Schwartz and Y. S. Yeh, "On the distribution function and moments of power sums with log-normal components," Bell Syst. Tech. J., vol. 61, no. 7, pp. 1441-1462, Sep. 1982.

[38] N. C. Beaulieu and Q. Xie, "An optimal lognormal approximation to lognormal sum distributions," IEEE Trans. Veh. Technol., vol. 53, no. 2, pp. 479-489, Mar. 2004.

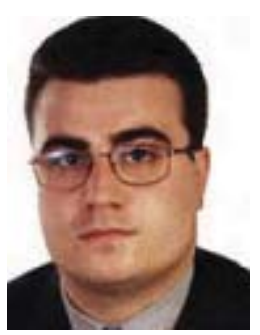

Marco Di Renzo (S'05-AM'07-M'09) was born in L'Aquila, Italy, in 1978. He received the Laurea (cum laude) and the Ph.D. degrees in Electrical and Information Engineering from the Department of Electrical and Information Engineering, University of L'Aquila, Italy, in April 2003 and January 2007, respectively. From August 2002 to January 2008, he was with the Center of Excellence in Research DEWS, University of L'Aquila, Italy. From February 2008 to April 2009, he was a tenured Research Associate with the Telecommunications Technological Center of Catalonia, Barcelona, Spain. From May 2009 to December 2009, he was a Research Fellow with the Institute for Digital Communications (IDCOM), The University of Edinburgh, Edinburgh, Scotland, United Kingdom (UK). Since January 2010, ha has been a Researcher (Chargé de Recherche), with the French National Center for Scientific Research (CNRS), Laboratory for Signals and Systems (LSS), École Supérieure d'Électricité (SUPÉLEC), Paris, France.

In December 2004, he was co-founder of WEST Aquila s.r.l. (Wireless Embedded Systems Technologies L'Aquila), an R\&D Spin-Off of the Department of Electrical and Information Engineering, and the Center of Excellence in Research DEWS, where he currently holds the position of Senior Research Engineer in wireless communications. In 2006, he was a Visiting Scholar with the Mobile and Portable Radio Research Group in the Bradley Department of Electrical and Computer Engineering, Virginia Polytechnic Institute and State University, USA. His main research interests are in the area of wireless communication theory and wireless communication systems design.

Dr. Di Renzo was awarded the "mention" for the outstanding five-year (1997-2003) academic career from the University of L'Aquila, Italy; a three-year Ph.D. fellowship (ranked 1st) from the Department of Electrical and Information Engineering, University of L'Aquila, Italy, and THALES Communications s.p.a, Land and Joint Systems Division of Advanced Studies, Chieti, Italy; a research fellowship from the Center of Excellence in Research DEWS, University of L'Aquila, Italy, for conducting research in the USA; and a two-year personal Spanish "Torres Quevedo" national grant (PTQ-0801-06437) from the "Ministerio de Ciencia e Innovacion" for his research on ultra wide band systems and cooperative localization for wireless sensor networks.

Dr. Di Renzo is a Member of the IEEE and IEEE Communications Society, and serves as a reviewer for transactions journals and international conferences. He is currently serving as Publicity Chair of the 2010 International Conference on Mobile Lightweight Wireless Systems (Mobilight). He also serves as a Technical Program Committee (TPC) member and Session Chairman of several international conferences in communications. 


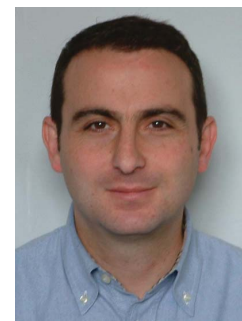

Fabio Graziosi (S'96-M'97) was born in L'Aquila, Italy, in 1968. He received the Laurea degree (cum laude) and the Ph.D. degree in electronic engineering from the University of L'Aquila, L'Aquila, in 1993 and 1997, respectively.

Since February 1997, he has been with the Department of Electrical Engineering, University of L'Aquila, where he is currently an Associate Professor. $\mathrm{He}$ is a member of the Executive Committee, Center of Excellence Design methodologies for Embedded controllers, Wireless interconnect and System-on-chip (DEWS), University of L'Aquila, and the Executive Committee, Consorzio Nazionale Interuniversitario per le Telecomunicazioni (CNIT). $\mathrm{He}$ is also the Chairman of the Board of Directors of WEST Aquila s.r.l., a spin-off R\&D company of the University of L'Aquila and the Center of Excellence DEWS. He is involved in major national and European research programs in the field of wireless systems and he has been a reviewer for major technical journals and international conferences in communications. $\mathrm{He}$ also serves as a Technical Program Committee (TPC) member and Session Chairman of several international conferences in communications. His current research interests are mainly focused on wireless communication systems with emphasis on wireless sensor networks, ultra wide band communication techniques, cognitive radio, and cooperative communications.

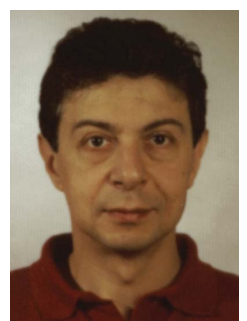

Fortunato Santucci (S'93-M'95-SM'00) was born in L'Aquila, Italy, in 1964. He received the Laurea and $\mathrm{Ph} . \mathrm{D}$. degrees in electronic engineering from the University of L'Aquila, L'Aquila, in 1989 and 1994, respectively.

In 1989, he was with Selenia Spazio S.p.A., Rome, Italy, where he was engaged in very small aperture terminals (VSAT) networks design. During 1991-1992, he was with the Solid State Electronics Institute (I.E.S.S.), National Research Council (C.N.R.), Rome, where he was involved in the research on superconductor receivers for millimeter wave satellite systems. Since 1994, he has been with the Department of Electrical Engineering, University of L'Aquila, where he is currently an Associate Professor. During 1996, he was a Visiting Researcher in the Department of Electrical and Computer Engineering, University of Victoria, Victoria, BC, Canada, where he researched on code division multiple access (CDMA) networks. He is a member of the Executive Committee, Center of Excellence Design Methodologies for Embedded Controllers, Wireless Interconnect and Systemon-Chip (DEWS), University of L'Aquila, and the Executive Committee, Consorzio Nazionale Interuniversitario per le Telecomunicazioni (CNIT). He has been a reviewer for several technical journal in telecommunications, and is currently an Editor for KLUWER TELECOMMUNICATIONS SYSTEMS. $\mathrm{He}$ has participated in various national and European research programs in wireless mobile communications and coordinates research programs funded by industrial partners. His current research interests include communication theory, access control, and radio resource management in wireless systems with special emphasis on technologies for networked embedded systems.

Dr. Santucci is currently an Editor of the IEEE TRANSACTIONS ON COMMUNICATIONS. He is also a member of the technical program committees (TPC) of several conferences in communications. He is a member of the Communications Theory Committee and a Session Chairman of various conferences. 